\title{
Singular behaviour of the electromagnetic field
}

\author{
C. Vrejoiut and R. Zus $\mathrm{g}$ \\ University of Bucharest, Department of Physics, \\ PO Box MG - 11, Bucharest-Magurele, RO - 077125, Romania
}

\begin{abstract}
The singularities of the electromagnetic field are derived to include all the point-like multipoles representing an electric charge and current distribution. Firstly derived in the static case, the result is generalized to the dynamic one. We establish a simple procedure for passing from the first, to the second case.
\end{abstract}

\section{Introduction}

In the cases of electrostatic and magnetostatic fields of point-like dipoles, one has the well-known procedure of introducing Dirac $\delta$-function terms for obtaining correct expressions of the electric and magnetic fields defined on the entire space. The corresponding field expressions take the following form [1]:

$$
\boldsymbol{E}_{\boldsymbol{p}}(\boldsymbol{r})=-\frac{1}{3 \varepsilon_{0}} \boldsymbol{p} \delta(\boldsymbol{r})+\frac{1}{4 \pi \varepsilon_{0}} \frac{3(\boldsymbol{\nu} \cdot \boldsymbol{p}) \boldsymbol{\nu}-\boldsymbol{p}}{r^{3}}=-\frac{1}{3 \varepsilon_{0}} \boldsymbol{p} \delta(\boldsymbol{r})+(\boldsymbol{E})_{r \neq 0}
$$

where $\boldsymbol{\nu}=\boldsymbol{r} / r$, and

$$
\boldsymbol{B}_{\boldsymbol{m}}(\boldsymbol{r})=\frac{2 \mu_{0}}{3} \boldsymbol{m} \delta(\boldsymbol{r})+\frac{\mu_{0}}{4 \pi} \frac{3(\boldsymbol{\nu} \cdot \boldsymbol{m}) \boldsymbol{\nu}-\boldsymbol{m}}{r^{3}}=\frac{2 \mu_{0}}{3} \boldsymbol{m} \delta(\boldsymbol{r})+(\boldsymbol{B})_{r \neq 0}
$$

In these equations, by $(\ldots)_{r \neq 0}$ we understand an expression in which the derivatives are calculated supposing $r \neq 0$, representing some well-known expressions of the fields. The expressions from equations (1) and (2) are introduced in Ref. [1] as conditions of compatibility with the average value of the electric or magnetic field over a spherical domain containing all the charges or currents inside. Another procedure for introducing equations (11) and (2) is based on an extension of the derivative $\partial_{i} \partial_{j} /(1 / r)$ to the entire space [2]:

$$
\partial_{i} \partial_{j} \frac{1}{r}=-\frac{4 \pi}{3} \delta_{i j} \delta(\boldsymbol{r})+\frac{3 \nu_{i} \nu_{j}-\delta_{i j}}{r^{3}} .
$$

A more pedagogical and suitable approach for understanding the origin of the difference between the electric and magnetic cases, is done in Ref. 3]. Refs. [4] and [5] contain generalizations of the equations (11) and (2) to the dynamic case for oscillating electric and magnetic dipoles.

$\ddagger$ E-mail : vrejoiu@fizica.unibuc.ro

$\S$ E-mail: roxana.zus@fizica.unibuc.ro 
The objective of the present paper is to establish the singularities of the electromagnetic field associated to a system of electric charges and currents assimilated with a point-like multipolar system. These singularities are established for an arbitrary multipolar order for both, the static and the dynamic cases.

In section 2, the procedure of separating the $\delta$-type singularities of the wave equation retarded solution is described. In the next section, based on the exterior solution of a given static electric charge and current distribution expressed in terms of electric and magnetic multipoles of this distribution, i.e. the multipole expansion of the electromagnetic field, we separate the $\delta$-type singularities of the fields $\boldsymbol{E}$ and $\boldsymbol{B}$. The calculation is based on the invariance of the static electromagnetic field to the substitution of the Cartesian tensors representing the multipole electric and magnetic moments by the corresponding symmetric trace free (STF) projections. In section 4 , the singularities in the dynamic case are established by employing also the invariance of the electromagnetic field to the substitution of multipole Cartesian moments by STF tensors, but this time, generally different from the corresponding STF projections.

In Appendix A, we give some furmulae for the lower order spatial derivatives of the spherical wave retarded solution used in the current exposition. In the second appendix, the reduction to the $\mathbf{S T F}$ tensors in the dynamic case and for low orders is presented.

We point out that the formalism presented in this issue has as mathematical basis the properties of the irreducible tensorial representations of the proper rotations group [6]. Although, for pedagogical and larger accessibility reasons, we give an explicit calculation based on the properties of the tensor contractions such that the procedure has a simple algebraic character. A counterpart of the procedure used in the present paper could be represented by the technique of the spherical function expansions and the cited issues can be a basis for such an approach.

\section{Some delta-function identities}

The treatment of some delta-function identities in Ref. [2] can be easily generalized to obtain the necessary delta-function identities in the dynamic case. Let us consider the solution $f(t-r / c) / r$ of the wave equation.

$$
\left(\Delta-\frac{1}{c^{2}} \frac{\partial^{2}}{\partial t^{2}}\right) \frac{f\left(t-\frac{r}{c}\right)}{r}=-4 \pi f(t) \delta(\boldsymbol{r}) .
$$

In the points different from the origin $O$, this function is a solution of the homogeneous wave equation. The multiple partial derivatives of this function are given, for $r \neq 0$, by the formula:

$$
\left(\partial_{i_{1}} \ldots \partial_{i_{n}} \frac{f(\tau)}{r}\right)_{r \neq 0}=\sum_{l=0}^{n} \frac{1}{c^{n-l} r^{l+1}} C_{i_{1} \ldots i_{n}}^{(n, l)} \frac{\partial^{n-l} f(\tau)}{\partial t^{n-l}}
$$

where $\tau=t-r / c$. The coefficients are symmetric in $i_{1}, \ldots, i_{n}$ and can be expressed as

$$
C_{i_{1} \ldots i_{n}}^{(n, l)}=\sum_{k=0}^{\left[\frac{n}{2}\right]} D_{k}^{(n, l)} \delta_{\left\{i_{1} i_{2}\right.} \ldots \delta_{i_{2 k-1} i_{2 k}} \nu_{2 k+1} \ldots \nu_{\left.i_{n}\right\}} .
$$


In the last equation, $[\beta]$ is the integer part of $\beta$ and $\boldsymbol{\nu}=\boldsymbol{r} / r$. By $A_{\left\{i_{1} \ldots i_{n}\right\}}$ we understand the sum over all the permutations of the symbols $i_{q}$ giving distinct terms. For the objective of the present paper, the coefficients $D$ in equation (6) can be calculated directly from the successive derivative operations. We give in appendix A the corresponding expressions for $n \leq 4$.

From equation (44), it is obvious that we have to consider the derivatives of $f(\tau) / r$ extended over the entire space as distributions (generalized functions) which contain singular distributions, as $\delta$-functions for example, as separate terms. Let be a function $F(\boldsymbol{r}, t)$ and suppose the existence of the integral of the product $F(\boldsymbol{r}, t) \phi(\boldsymbol{r})$, with $\phi(\boldsymbol{r})$ an arbitrary smooth function (a test function from the domain of the distributions), on the spherical region $\mathcal{D}_{R}$, with arbitrary radius $R$, delimited by the spherical surface $\Sigma_{R}$ with the center in $O$.

$$
\int_{\mathcal{D}_{R}} \mathrm{~d}^{3} x F(\boldsymbol{r}, t) \phi(\boldsymbol{r})=\lim _{\varepsilon \rightarrow 0} \int_{\varepsilon<r<R} \mathrm{~d}^{3} x F(\boldsymbol{r}, t) \phi(\boldsymbol{r})
$$

The integral can be expressed excluding from the domain $\mathcal{D}_{R}$ a spherical domain of radius $\varepsilon$ centered in $O$. Writing this last limit of integrals, we can interpret the function $F(\boldsymbol{r}, t)$ as a distribution defined by

$$
\left\langle(F(\boldsymbol{r}, t))_{r \neq 0}, \phi(\boldsymbol{r})\right\rangle=\lim _{\varepsilon \rightarrow 0} \int_{\mathcal{D}_{R} \backslash \mathcal{D}_{\varepsilon}} \mathrm{d}^{3} x(F(\boldsymbol{r}, t))_{r \neq 0} \phi(\boldsymbol{r}) .
$$

This distribution can be extended such that its support includes the point $O$. A new term $\theta(\varepsilon-r) F(\boldsymbol{r}, t)$ can be naturally introduced by the identity

$$
F_{\varepsilon}(\boldsymbol{r}, t)=\theta(\varepsilon-r) F(\boldsymbol{r}, t)+\theta(r-\varepsilon) F(\boldsymbol{r}, t),
$$

associated with the extension of the integral to the entire domain $\mathcal{D}_{R}$ :

$$
\langle F(\boldsymbol{r}, t), \phi(\boldsymbol{r})\rangle=\lim _{\varepsilon \rightarrow 0}\left[\int_{\mathcal{D}_{\varepsilon}} \mathrm{d}^{3} x F(\boldsymbol{r}, t) \phi(\boldsymbol{r})+\int_{\mathcal{D}_{R} \backslash \mathcal{D}_{\varepsilon}} \mathrm{d}^{3} x F(\boldsymbol{r}, t) \phi(\boldsymbol{r})\right] .
$$

Moreover, we suppose the existence of the integral (7) for the partial derivatives of $F$. Let us consider the partial derivative $\partial_{i} F(\boldsymbol{r}, t)$ and the problem of extending this function as a distribution. The definition (8) becomes:

$$
\begin{aligned}
\langle F(\boldsymbol{r}, t), \phi(\boldsymbol{r})\rangle=\lim _{\varepsilon \rightarrow 0}\left[\oint_{\Sigma_{\varepsilon}} \mathrm{d} S \nu_{i} F(\boldsymbol{r}, t) \phi(\boldsymbol{r})-\int_{\mathcal{D}_{\varepsilon}} \mathrm{d}^{3} x F(\boldsymbol{r}, t) \partial_{i} \phi(\boldsymbol{r})\right. \\
\left.+\int_{\mathcal{D}_{R} \backslash \mathcal{D}_{\varepsilon}} \mathrm{d}^{3} x \partial_{i} F(\boldsymbol{r}, t) \phi(\boldsymbol{r})\right],
\end{aligned}
$$

where $\Sigma_{\varepsilon}$ is the sphere of radius $\varepsilon$ centered in $O$ and the Gauss theorem was employed. Let us apply this definition to the derivative $\partial_{i} \partial_{j}(f(\tau) / r)$ and, for simplifying the notation, let

$$
D_{i_{1} \ldots i_{n}}(\boldsymbol{r}, t)=\partial_{i_{1}} \ldots \partial_{i_{n}} \frac{f(\tau)}{r},
$$

such that we can write

$$
\begin{aligned}
\left(D_{i j}, \phi\right)=\lim _{\varepsilon \rightarrow 0} & {\left[\oint_{\Sigma_{\varepsilon}} \mathrm{d} S \nu_{i} \partial_{j} \frac{f(\tau)}{r} \phi(\boldsymbol{r})-\int_{\mathcal{D}_{\varepsilon}} \mathrm{d}^{3} x \partial_{j} \frac{f(\tau)}{r} \partial_{i} \phi(\boldsymbol{r})\right.} \\
& \left.+\int_{\mathcal{D}_{R} \backslash \mathcal{D}_{\varepsilon}} \mathrm{d}^{3} x\left(\partial_{i} \partial_{j} \frac{f(\tau)}{r}\right) \phi(\boldsymbol{r})\right] .
\end{aligned}
$$


Since the last integral on the domain $\mathcal{D}_{R} \backslash \mathcal{D}_{\varepsilon}$ represents the distributions associated with the $F$ - expressions for $r \neq 0$ and, in the case of the electromagnetic field, will be the well-known expressions of the multipole expansions, in the following we consider only that part of $\left\langle D_{i j}\right\rangle$ containing singular distributions with point-like support i.e., actually, the difference

$$
\begin{aligned}
\left\langle\left(D_{i j}\right)_{(0)}, \phi\right\rangle & =\left\langle D_{i j}, \phi\right\rangle-\lim _{\varepsilon \rightarrow 0} \int_{\mathcal{D}_{R} \backslash \mathcal{D}_{\varepsilon}} \mathrm{d}^{3} x\left(\partial_{i} \partial_{j} \frac{f(\tau)}{r}\right) \phi(\boldsymbol{r}) \\
& =\lim _{\varepsilon \rightarrow 0}\left[\oint_{\Sigma_{\varepsilon}} \mathrm{d} S \nu_{i} \partial_{j} \frac{f(\tau)}{r} \phi(\boldsymbol{r})-\int_{\mathcal{D}_{\varepsilon}} \mathrm{d}^{3} x \partial_{j} \frac{f(\tau)}{r} \partial_{i} \phi(\boldsymbol{r})\right]
\end{aligned}
$$

By $D_{\left(\boldsymbol{r}_{0}\right)}$ we denote a distribution having as support the point given by the vector $\boldsymbol{r}_{0}$. In the first limit, we make use of equation (5) and of the expression of the coefficients $C_{i}^{(1, l)}$ from Appendix A:

$$
\lim _{\varepsilon \rightarrow 0} \oint_{\Sigma_{\varepsilon}} \mathrm{d} S \nu_{i} \partial_{j} \frac{f(\tau)}{r} \phi(\boldsymbol{r})=-\lim _{\varepsilon \rightarrow 0} \oint_{\Sigma_{\varepsilon}} \mathrm{d} S \nu_{i} \nu_{j}\left[\frac{1}{c r} \dot{f}(\tau)+\frac{1}{r^{2}} f(\tau)\right] \phi(\boldsymbol{r}) .
$$

Inserting the Taylor series of the function $\phi(\boldsymbol{r})$ and since on the sphere $r=\varepsilon$, we can write

$$
\begin{aligned}
& \lim _{\varepsilon \rightarrow 0} \oint_{\Sigma_{\varepsilon}} \mathrm{d} S \nu_{i} \partial_{j} \frac{f(\tau)}{r} \phi(\boldsymbol{r}) \\
& =-4 \pi \lim _{\varepsilon \rightarrow 0} \int \mathrm{d} \Omega(\boldsymbol{\nu}) \nu_{i} \nu_{j}\left[\frac{\varepsilon}{c} \dot{f}\left(\tau_{\varepsilon}\right)+f\left(\tau_{\varepsilon}\right)\right]\left[\phi(0)+\varepsilon \nu_{k}\left(\partial_{k} \phi\right)_{0}+\ldots\right]
\end{aligned}
$$

where $\tau_{\varepsilon}=t-\varepsilon / c$. Let us introduce the angular average:

$$
\langle g(\boldsymbol{\nu})\rangle=\frac{1}{4 \pi} \int g(\boldsymbol{\nu}) \mathrm{d} \Omega(\boldsymbol{\nu}) .
$$

Particularly, we have the well-known formula [7]:

$$
\left\langle\nu_{i_{1}} \ldots \nu_{i_{n}}\right\rangle=\left\{\begin{array}{cc}
0, & n=2 k+1, \\
\frac{1}{(n+1) ! !} \delta_{\left\{i_{1} i_{2}\right.} \ldots \delta_{\left.i_{n-1} i_{n}\right\}}, & n=2 k, \quad k=0,1, \ldots
\end{array}\right.
$$

Excepting the term containing the product $f\left(\tau_{\varepsilon}\right) \phi(0)$, all the terms in equation (12) are proportional to positive powers of $\varepsilon$ and, consequently, vanish with $\varepsilon \rightarrow 0$ such that

$$
\begin{aligned}
\lim _{\varepsilon \rightarrow 0} \oint_{\Sigma_{\varepsilon}} \mathrm{d} S \nu_{i} \partial_{j} \frac{f(\tau)}{r} \phi(\boldsymbol{r}) & =-4 \pi\left\langle\nu_{i} \nu_{j}\right\rangle f(t) \phi(0) \\
& =-\frac{4 \pi}{3} \delta_{i j} f(t) \phi(0)=-\frac{4 \pi}{3} \delta_{i j} f(t)\langle\delta(\boldsymbol{r}), \phi(\boldsymbol{r})\rangle .
\end{aligned}
$$

Considering the second integral in the right-hand side of equation (111), we can write

$$
\begin{aligned}
& \lim _{\varepsilon \rightarrow 0} \int_{\mathcal{D}_{\varepsilon}} \mathrm{d}^{3} x \partial_{j} \frac{f(\tau)}{r} \partial_{i} \phi(\boldsymbol{r}) \\
= & -\lim _{\varepsilon \rightarrow 0} \int_{0}^{\varepsilon} r^{2} \mathrm{~d} r \int \mathrm{d} \Omega(\boldsymbol{\nu}) \nu_{j}\left[\frac{1}{c r} \dot{f}(\tau)+\frac{1}{r^{2}} f(\tau)\right]\left[\left(\partial_{i} \phi\right)_{0}+r \nu_{k}\left(\partial_{i} \partial_{k} \phi\right)_{0}+\ldots\right]=0
\end{aligned}
$$

such that finally

$$
\left(\partial_{i} \partial_{j} \frac{f(\tau)}{r}\right)_{(0)}=-\frac{4 \pi}{3} \delta_{i j} f(t) \delta(\boldsymbol{r})
$$


In the static case, $f(t)=1$, equation (16) becomes equation (3) from Ref. [2] after adding the regular distribution represented by the last integral from equation (10).

Let us consider the distribution $D_{i j k}$. Considering only the part having $O$ as support,

$$
\left(\left(D_{i j k}\right)_{(0)}, \phi\right)=\lim _{\varepsilon \rightarrow 0}\left[\oint_{\Sigma_{\varepsilon}} \mathrm{d} S \nu_{i} \partial_{j} \partial_{k} \frac{f(\tau)}{r} \phi(\boldsymbol{r})-\int_{\mathcal{D}_{\varepsilon}} \mathrm{d}^{3} x\left(\partial_{j} \partial_{k} \frac{f(\tau)}{r}\right) \partial_{i} \phi(\boldsymbol{r})\right] .
$$

The surface integral becomes:

$$
\begin{gathered}
\lim _{\varepsilon \rightarrow 0} \oint_{\Sigma_{\varepsilon}} \mathrm{d} S \nu_{i} \partial_{j} \partial_{k} \frac{f(\tau)}{r} \phi(\boldsymbol{r})=\lim _{\varepsilon \rightarrow 0} \oint_{\Sigma_{\varepsilon}} \mathrm{d} S \nu_{i}\left[\frac{1}{c^{2} r} \nu_{j} \nu_{k} \ddot{f}(\tau)+\frac{1}{c r^{2}}\left(3 \nu_{j} \nu_{k}-\delta_{j k}\right) \dot{f}(\tau)\right. \\
\left.+\frac{1}{r^{3}}\left(3 \nu_{j} \nu_{k}-\delta_{j k}\right) f(\tau)\right] \phi(\boldsymbol{r})
\end{gathered}
$$

and, introducing the Taylor series for $\phi(\boldsymbol{r})$,

$$
\begin{aligned}
& \lim _{\varepsilon \rightarrow 0} \oint_{\Sigma_{\varepsilon}} \mathrm{d} S \nu_{i} \partial_{j} \partial_{k} \frac{f(\tau)}{r} \phi(\boldsymbol{r})=4 \pi \lim _{\varepsilon \rightarrow 0}\left\langle\left[\frac{\varepsilon}{c^{2}} \nu_{i} \nu_{j} \nu_{k} \ddot{f}\left(\tau_{\varepsilon}\right)\right.\right. \\
& \left.+\frac{1}{c}\left(3 \nu_{i} \nu_{j} \nu_{k}-\nu_{i} \delta_{j k} \dot{f}\left(\tau_{\varepsilon}\right)+\frac{1}{\varepsilon}\left(3 \nu_{i} \nu_{j} \nu_{k}-\nu_{i} \delta_{j k}\right) f\left(\tau_{\varepsilon}\right)\right]\left[\phi(0)+\varepsilon \nu_{l}\left(\partial_{l} \phi\right)_{0}+\ldots\right]\right\rangle .
\end{aligned}
$$

All the terms containing the products $\ddot{f}\left(\tau_{\varepsilon}\right) \phi(0)$ and $\dot{f}\left(\tau_{\varepsilon}\right) \phi(0)$ give null results being multiplied by averages of three factors $\nu$. The other terms containing the factors $\ddot{f}\left(\tau_{\varepsilon}\right)$ and $\dot{f}\left(\tau_{\varepsilon}\right)$ give also null results being proportional to positive powers of $\varepsilon$. The term provided by the product $f\left(\tau_{\varepsilon}\right) \phi(0)$ contains the factor $\varepsilon^{-1}$, but it has also a null limit since $\left\langle 3 \nu_{i} \nu_{j} \nu_{k}-\nu_{i} \delta_{j k}\right\rangle=0$. Only the product $f\left(\tau_{\varepsilon}\right)\left(\partial_{l} \phi\right)_{0}$ has a factor independent of $\varepsilon$ having a limit different from zero.

Therefore,

$$
\begin{aligned}
\lim _{\varepsilon \rightarrow 0} \oint_{\Sigma_{\varepsilon}} \mathrm{d} S \nu_{i} \partial_{j} \partial_{k} \frac{f(\tau)}{r} \phi(\boldsymbol{r}) & =4 \pi\left(3 \nu_{i} \nu_{j} \nu_{k} \nu_{l}-\nu_{i} \nu_{l} \delta_{j k}\right) f(t)\left(\partial_{l} \phi\right)_{0} \\
& =4 \pi\left(\frac{1}{5} \delta_{\{i j} \delta_{k l\}}-\frac{1}{3} \delta_{i l} \delta_{j k}\right) f(t)\left(\partial_{l} \phi\right)_{0}
\end{aligned}
$$

Concerning the integral on $\mathcal{D}_{\varepsilon}$ from equation (17), we have to observe that, beginning from this derivative order, there is a non-zero contribution for $\varepsilon \rightarrow 0$ [2. Indeed, introducing equation (16) in equation (17) and observing that the term $\left(\partial_{j} \partial_{k}(f(\tau) / r)\right)_{r \neq 0}$ gives a null contribution to the limit for $\varepsilon \rightarrow 0$, we can write

$$
-\lim _{\varepsilon \rightarrow 0} \int_{\mathcal{D}_{\varepsilon}} \mathrm{d}^{3} x \partial_{j} \partial_{k} \frac{f(\tau)}{r} \partial_{i} \phi(\boldsymbol{r})=\frac{4 \pi}{3} \int_{\mathcal{D}_{\varepsilon}} \mathrm{d}^{3} x f(t) \delta_{j k} \delta(\boldsymbol{r}) \partial_{i} \phi(\boldsymbol{r})=\frac{4 \pi}{3} f(t) \delta_{j k}\left(\partial_{i} \phi\right)_{0} .
$$

Finally, equations (17), (18) and (19) give

$$
\left(D_{i j k}\right)_{(0)}=-\frac{4 \pi}{5} f(t) \delta_{\{i j} \partial_{k\}} \delta(\boldsymbol{r}) \text {. }
$$

For the static case, $f(t)=1$; this result corresponds to equation (4) from Ref. [2].

Obviously, this procedure becomes very complicated for higher order derivatives. Fortunately, for the electromagnetic field some invariance properties allow a considerable simplification of such calculations. 


\section{Singularities of the electromagnetic field: the static case}

Let us consider the multipole expansions of the electrostatic and magnetostatic fields. Given an electric charge and current distribution with support included in the domain $\mathcal{D}$, the scalar potential is expressed in the exterior of this domain by the following multipolar series:

$$
\Phi(\boldsymbol{r})=\frac{1}{4 \pi \varepsilon_{0}} \sum_{n \geq 0} \frac{(-1)^{n}}{n !} \partial_{i_{1}} \ldots \partial_{i_{n}} \frac{\mathrm{P}_{i_{1} \ldots i_{n}}}{r}=\frac{1}{4 \pi \varepsilon_{0}} \sum_{n \geq 0} \frac{(-1)^{n}}{n !} \nabla^{n} \| \frac{\mathbf{P}^{(n)}}{r} .
$$

In this expansion, the coordinate system origin $O$ is supposed in $\mathcal{D}$ and $\mathbf{P}^{(n)}$ is the $n$-th order electric multipolar moment defined by the Cartesian components in the general dynamic case:

$$
\mathrm{P}_{i_{1} \ldots i_{n}}(t)=\int_{\mathcal{D}} \mathrm{d}^{3} x x_{i_{1}} \ldots x_{i_{n}} \rho(\boldsymbol{r}, t): \mathbf{P}^{(n)}(t)=\int_{\mathcal{D}} \mathrm{d}^{3} x \boldsymbol{r}^{n} \rho(\boldsymbol{r}, t) .
$$

In equation (22) we employed the following notation for tensorial contractions:

$$
\left(\mathbf{A}^{(n)} \| \mathbf{B}^{(m)}\right)_{i_{1} \cdots i_{|n-m|}}= \begin{cases}A_{i_{1} \cdots i_{n-m} j_{1} \cdots j_{m}} B_{j_{1} \cdots j_{m}} & , n>m \\ A_{j_{1} \cdots j_{n}} B_{j_{1} \cdots j_{n}} & , n=m \\ A_{j_{1} \cdots j_{n}} B_{j_{1} \cdots j_{n} i_{1} \cdots i_{m-n}} & , n<m\end{cases}
$$

For the vector potential in the exterior of the domain $\mathcal{D}$, we have

$$
\begin{aligned}
\boldsymbol{A}(\boldsymbol{r}) & =\frac{\mu_{0}}{4 \pi} \sum_{n \geq 1} \frac{(-1)^{n-1}}{n !} \nabla \times\left(\boldsymbol{\nabla}^{n-1} \| \mathbf{M}^{(n)}\right) \\
& =\frac{\mu_{0}}{4 \pi} \boldsymbol{e}_{i} \varepsilon_{i j k} \partial_{j} \sum_{n \geq 1} \frac{(-1)^{n-1}}{n !} \partial_{i_{1}} \ldots \partial_{i_{n-1}} \frac{\mathrm{M}_{i_{1} \ldots i_{n-1} k}}{r},
\end{aligned}
$$

where $\mathbf{M}^{(n)}$ is the magnetic $n$-th order moment defined by the Cartesian components [8]:

$$
\mathrm{M}_{i_{1} \ldots i_{n}}(t)=\frac{n}{n+1} \int_{\mathcal{D}} \mathrm{d}^{3} x x_{i_{1}} \ldots x_{i_{n-1}}(\boldsymbol{r} \times \boldsymbol{J}(\boldsymbol{r}, t))_{i_{n}},
$$

or, with tensorial notation:

$$
\mathbf{M}^{(n)}(t)=\frac{n}{n+1} \int_{\mathcal{D}} \mathrm{d}^{3} x \boldsymbol{r}^{n} \times \boldsymbol{J}(\boldsymbol{r}, t) .
$$

For the multipole expansion of the electric field $\boldsymbol{E}(\boldsymbol{r})=-\boldsymbol{\nabla} \Phi(\boldsymbol{r})$, equation (22) becomes:

$$
\boldsymbol{E}(\boldsymbol{r})=\frac{1}{4 \pi \varepsilon_{0}} \sum_{n \geq 1} \frac{(-1)^{n-1}}{n !} \nabla^{n+1} \| \frac{\mathbf{P}^{(n)}}{r}=\frac{1}{4 \pi \varepsilon_{0}} \boldsymbol{e}_{i} \sum_{n \geq 1} \frac{(-1)^{n-1}}{n !} \partial_{i} \partial_{i_{1}} \ldots \partial_{i_{n}} \frac{\mathrm{P}_{i_{1} \ldots i_{n}}}{r},
$$

where, for simplicity, the electric charged system is considered neutral $(Q=0)$.

The corresponding expansion of the magnetic field $\boldsymbol{B}(\boldsymbol{r})=\boldsymbol{\nabla} \times \boldsymbol{A}(\boldsymbol{r})$ is given by

$$
\begin{aligned}
\boldsymbol{B}(\boldsymbol{r}) & =\frac{\mu_{0}}{4 \pi} \boldsymbol{\nabla} \times \sum_{n \geq 1} \frac{(-1)^{n-1}}{n !} \boldsymbol{\nabla} \times\left(\boldsymbol{\nabla}^{n-1} \| \frac{\mathbf{M}^{(n)}}{r}\right) \\
& =\frac{\mu_{0}}{4 \pi} \sum_{n \geq 1} \frac{(-1)^{n-1}}{n !}\left[\boldsymbol{\nabla} \cdot\left(\boldsymbol{\nabla}^{n} \| \frac{\mathbf{M}^{n)}}{r}\right)-\Delta\left(\boldsymbol{\nabla}^{n-1} \| \frac{\mathbf{M}^{(n)}}{r}\right)\right] .
\end{aligned}
$$


We have to search the singularities of $\boldsymbol{E}$ and $\boldsymbol{B}$ given by equations (27) and (28). It appears that cumbersome calculations are involved for higher $n$ if we apply the formulae for derivatives of an arbitrary function $f(\tau) / r$ as in the previous section. However, we can employ an invariance property of the electromagnetic static field to the substitutions of all moments $\mathbf{P}^{(n)}$ and $\mathbf{M}^{(n)}$ for all $n$ by their corresponding symmetric and trace-free STF projections $\mathcal{P}^{(n)}$ and $\mathcal{M}^{(n)}$ [9, 10, 11]. Retaining the notation $\boldsymbol{p}$ and $\boldsymbol{m}$ for the first order moments, this invariance stands for the invariance of the electromagnetic field, in the static case, to the following substitutions:

$$
\begin{aligned}
& \boldsymbol{p}, \mathbf{P}^{(2)}, \mathbf{P}^{(3)}, \ldots \quad \rightarrow \boldsymbol{p}, \mathcal{P}^{(2)}, \mathcal{P}^{(3)} \ldots ; \\
& \boldsymbol{m}, \mathbf{M}^{(2)}, \mathbf{M}^{(3)}, \ldots \rightarrow \boldsymbol{m}, \mathcal{M}^{(2)}, \mathcal{M}^{(3)} \ldots
\end{aligned}
$$

These $\mathbf{S T F}$ tensors can be expressed by the following formulae:

$$
\begin{aligned}
\mathcal{P}_{i_{1} \ldots i_{n}} & =\frac{(-1)^{n}}{(2 n-1) ! !} \int_{\mathcal{D}} \mathrm{d}^{3} x \rho(\boldsymbol{r}) \boldsymbol{r}^{2 n+1} \partial_{i_{1}} \ldots \partial_{i_{n}} \frac{1}{r}, \\
\mathcal{M}_{i_{1} \ldots i_{n}} & =\frac{(-1)^{n+1}}{(n+1)(2 n-1) ! !} \sum_{\lambda=1}^{n} \int_{\mathcal{D}} \mathrm{d}^{3} x \boldsymbol{r}^{2 n+1}(\boldsymbol{J} \times \nabla)_{i_{\lambda}} \partial_{i_{1} \ldots i_{n}}^{(\lambda)} \frac{1}{r},
\end{aligned}
$$

which, actually, differ from the projections by numerical factors. Here,

$$
\partial_{i_{1} \ldots i_{n}}^{(\lambda)}=\partial_{i_{1}} \ldots \partial_{i_{\lambda-1}} \partial_{i_{\lambda+1}} \ldots \partial_{i_{n}} .
$$

Let us consider the delta-singularity corresponding to the electric dipolar field:

$$
\boldsymbol{E}_{(0)}^{(1)}=\frac{1}{4 \pi \varepsilon_{0}} \boldsymbol{e}_{i} \partial_{i} \partial_{j} \frac{p_{j}}{r}=-\frac{1}{3 \varepsilon_{0}} \boldsymbol{p} \delta(\boldsymbol{r}),
$$

a result obtained applying directly equation (16) for $f(t)=p_{j}$. Concerning the 4-polar term from $\boldsymbol{E}$, we consider firstly the expansion (27) expressed by the primitive moments $\mathbf{P}^{(n)}$ :

$$
\boldsymbol{E}^{(2)}(\boldsymbol{r})=-\frac{1}{8 \pi \varepsilon_{0}} \boldsymbol{e}_{i} \partial_{i} \partial_{j} \partial_{k} \frac{\mathrm{P}_{j k}}{r}
$$

and from equation (21):

$$
\begin{aligned}
\boldsymbol{E}_{(0)}^{(2)} & =\frac{1}{10 \varepsilon_{0}} \boldsymbol{e}_{i} \mathrm{P}_{j k} \delta_{\{i j} \partial_{k\}} \delta(\boldsymbol{r})=\frac{1}{10 \varepsilon_{0}} \boldsymbol{e}_{i} \mathrm{P}_{j k}\left(\delta_{i j} \partial_{k} \delta(\boldsymbol{r})+\delta_{i k} \partial_{j} \delta(\boldsymbol{r})+\delta_{j k} \partial_{i} \delta(\boldsymbol{r})\right) \\
& =\frac{1}{10 \varepsilon_{0}} \boldsymbol{e}_{i}\left(2 \mathrm{P}_{i j} \partial_{j} \delta(\boldsymbol{r})+\mathrm{P}_{j j} \partial_{i} \delta(\boldsymbol{r})\right)=\frac{1}{5 \varepsilon_{0}} \boldsymbol{e}_{i}\left(\mathrm{P}_{i j} \partial_{j} \delta(\boldsymbol{r})+\frac{1}{2} \mathrm{P}_{j j} \partial_{i} \delta(\boldsymbol{r})\right) .
\end{aligned}
$$

Note that $\mathbf{P}^{(2)}$ is symmetric.

The substitution $\mathrm{P}_{i j} \rightarrow \mathcal{P}_{i j}$ in equation (33) gives

$$
\boldsymbol{E}_{(0)}^{(2)}=\frac{1}{5 \varepsilon_{0}} \boldsymbol{e}_{i} \mathcal{P}_{i j} \partial_{j} \delta(\boldsymbol{r})=\frac{1}{5 \varepsilon_{0}} \mathcal{P}^{(2)} \| \boldsymbol{\nabla} \delta(\boldsymbol{r}),
$$

since $\boldsymbol{P}^{(2)}$ is trace-less $\left(\mathcal{P}_{j j}=0\right)$.

From this simple example, it becomes obvious that it is more simple providing formulae for the delta-singularities directly for STF tensors. Let us consider the 4-pole approximation using the definition

$$
\begin{aligned}
\left\langle\boldsymbol{E}_{(0)}^{(2)}, \phi\right\rangle & =-\frac{1}{8 \pi \varepsilon_{0}} \lim _{\varepsilon \rightarrow 0} \boldsymbol{e}_{i} \int_{\mathcal{D}_{\varepsilon}} \mathrm{d}^{3} x\left(\partial_{i} \partial_{j} \partial_{k} \frac{\mathcal{P}_{j k}}{r}\right) \phi(\boldsymbol{r}) \\
& =-\frac{1}{8 \pi \varepsilon_{0}} \boldsymbol{e}_{i} \lim _{\varepsilon \rightarrow 0}\left[\oint_{\Sigma_{\varepsilon}} \mathrm{d} S \mathcal{P}_{j k} \nu_{i}\left(\partial_{j} \partial_{k} \frac{1}{r}\right) \phi(\boldsymbol{r})-\int_{\mathcal{D}_{\varepsilon}} \mathrm{d}^{3} x \mathcal{P}_{j k} \partial_{j} \partial_{k} \frac{1}{r} \partial_{i} \phi(\boldsymbol{r})\right]
\end{aligned}
$$


In the last integral over $\mathcal{D}_{\varepsilon}$ from equation (35), we can introduce equation (16) obtaining

$$
\begin{aligned}
& \lim _{\varepsilon \rightarrow 0} \int_{\mathcal{D}_{\varepsilon}} \mathrm{d}^{3} x \mathcal{P}_{j k}\left[-\frac{4 \pi}{3} \delta_{j k} \delta(\boldsymbol{r})+\left(\partial_{j} \partial_{k} \frac{1}{r}\right)_{r \neq 0}\right] \partial_{i} \phi(\boldsymbol{r})=\lim _{\varepsilon \rightarrow 0} \int_{\mathcal{D}_{\varepsilon}} \mathrm{d}^{3} x \mathcal{P}_{j k}\left(\partial_{j} \partial_{k} \frac{1}{r}\right)_{r \neq 0} \partial_{i} \phi(\boldsymbol{r}) \\
& =\lim _{\varepsilon \rightarrow 0} \int_{\mathcal{D}_{\varepsilon}} \mathrm{d}^{3} x \mathcal{P}_{j k} \frac{1}{r^{3}}\left(3 \nu_{j} \nu_{k}-\delta_{j k}\right) \partial_{i} \phi(\boldsymbol{r})=3 \lim _{\varepsilon \rightarrow 0} \int_{\mathcal{D}_{\varepsilon}} \mathrm{d}^{3} x \frac{1}{r^{3}} \mathcal{P}_{j k} \nu_{j} \nu_{k} \partial_{i} \phi(\boldsymbol{r}) \\
& =3 \lim _{\varepsilon \rightarrow 0} \int_{0}^{\varepsilon} \frac{\mathrm{d} r}{r} \oint \mathrm{d} \Omega \mathcal{P}_{j k} \nu_{j} \nu_{k}\left[\left(\partial_{i} \phi\right)_{0}+r \nu_{l}\left(\partial_{i} \partial_{l} \phi\right)_{0}+\ldots\right]=0,
\end{aligned}
$$

since the term $\mathcal{P}_{j k} \nu_{j} \nu_{k}\left(\partial_{i} \phi\right)_{0}$ provides the expression $\mathcal{P}_{j k} \delta_{j k}=0$ and for the other terms from the $\partial_{i} \phi$ series has null limits containing, after integration over $r$, positive powers of $\varepsilon$. The same result can be obtained by a repetition of the integration by parts in the last volume integral. Therefore, we can write

$$
\begin{aligned}
\left\langle\boldsymbol{E}_{(0)}^{(2)}, \phi\right\rangle & =-\frac{1}{8 \pi \varepsilon_{0}} \boldsymbol{e}_{i} \lim _{\varepsilon \rightarrow 0} \oint_{\Sigma_{\varepsilon}} \mathrm{d} S \mathcal{P}_{j k} \nu_{i}\left(\partial_{j} \partial_{k} \frac{1}{r}\right) \phi(\boldsymbol{r}) \\
& \left.=-\frac{1}{2 \varepsilon_{0}} \boldsymbol{e}_{i} \lim _{\varepsilon \rightarrow 0} \mathcal{P}_{j k} \frac{1}{\varepsilon}\left\langle\nu_{i}\left(3 \nu_{j} \nu_{k}-\delta_{j k}\right)\left(\phi(0)+\varepsilon \nu_{l}\left(\partial_{l} \phi\right)_{0}+\ldots\right)\right)\right\rangle \\
& \left.=-\frac{1}{2 \varepsilon_{0}} \boldsymbol{e}_{i} \mathcal{P}_{j k}\left\langle 3 \nu_{i} \nu_{j} \nu_{k} \nu_{l}\right\rangle\left(\partial_{l} \phi\right)\right)_{0}=-\frac{1}{10 \varepsilon_{0}} \boldsymbol{e}_{i} \mathcal{P}_{j k}\left(\delta_{\{i j} \delta_{k l\}}\right)\left(\partial_{l} \phi\right)_{0} \\
& =-\frac{1}{5 \varepsilon_{0}} \boldsymbol{P}^{(2)} \|(\boldsymbol{\nabla} \phi)_{0},
\end{aligned}
$$

i.e. the result (34).

Let us consider the general terms from the series representing the electric and magnetic fields in equations (27) and (28) with the substitutions (29) performed. Denoting by $\mathbf{T}^{(n)}$ an $n$-th order STF tensor, we observe the presence in both equations of an expression of the form

$$
\boldsymbol{\nabla}\left(\nabla^{n} \| \frac{\mathrm{T}^{(n)}}{r}\right)=\boldsymbol{e}_{i} \partial_{i} \partial_{i_{1}} \ldots \partial_{i_{n}} \frac{\mathrm{T}_{i_{1} \ldots i_{n}}}{r} .
$$

Searching the extension of this function to the entire space, we introduce the distribution $\boldsymbol{I}\left(\mathrm{T}^{(n)}\right)$ by

$$
\left\langle\boldsymbol{I}\left(\mathrm{T}^{(n)}\right), \phi\right\rangle=\lim _{\varepsilon \rightarrow 0} \int_{\mathcal{D}_{\varepsilon}} \mathrm{d}^{3} x \nabla\left(\nabla^{n} \frac{\mathbf{T}^{(n)}}{r}\right) .
$$

Writing the last equation as

$$
\left\langle\boldsymbol{I}\left(\mathrm{T}^{(n)}\right), \phi\right\rangle=\lim _{\varepsilon \rightarrow 0}\left[\oint_{\Sigma_{\varepsilon}} \mathrm{d} S \boldsymbol{\nu}\left(\boldsymbol{\nabla}^{n} \| \frac{\mathbf{T}^{(n)}}{r}\right) \phi(\boldsymbol{r})-\int_{\mathcal{D}_{\varepsilon}} \mathrm{d}^{3} x\left(\boldsymbol{\nabla}^{n} \| \frac{\mathbf{T}^{(n)}}{r}\right) \boldsymbol{\nabla} \phi(\boldsymbol{r})\right],
$$

we can consider separately the limit of the surface integral:

$$
\begin{aligned}
\left\langle\boldsymbol{I}_{\sigma}\left(\mathbf{T}^{(n)}\right), \phi\right\rangle & =\lim _{\varepsilon \rightarrow 0} \oint_{\Sigma_{\varepsilon}} \mathrm{d} S \boldsymbol{\nu}\left(\boldsymbol{\nabla}^{n} \| \frac{\mathbf{T}^{(n)}}{r}\right) \phi(\boldsymbol{r}) \\
& =\boldsymbol{e}_{i} \lim _{\varepsilon \rightarrow 0} \oint_{\Sigma_{\varepsilon}} \mathrm{d} S \nu_{i} \mathrm{~T}_{i_{1} \ldots i_{n}}\left(\partial_{i_{1}} \ldots \partial_{i_{n}} \frac{1}{r}\right) \phi(\boldsymbol{r}) .
\end{aligned}
$$

Applying equation (5) and the Taylor series of $\phi(\boldsymbol{r})$,

$$
\begin{aligned}
&\left\langle\boldsymbol{I}_{\sigma}\left(\mathbf{T}^{(n)}\right), \phi\right\rangle=4 \pi \boldsymbol{e}_{i} \lim _{\varepsilon \rightarrow 0} \sum_{\alpha=0}^{\infty} \frac{\varepsilon^{\alpha-n+1}}{\alpha !} \mathbf{T}_{i_{1} \ldots i_{n}}\left\langle\nu_{i} C_{i_{1} \ldots i_{n}}^{(n, n)} \nu_{i_{n+1}} \ldots \nu_{i_{n+\alpha}}\right\rangle \\
& \times\left(\partial_{i_{n+1}} \ldots \partial_{i_{n+\alpha}} \phi\right)_{0} .
\end{aligned}
$$


Let us evaluate the tensorial contraction which is present in the general term from the previous series:

$$
\mathbf{T}_{i_{1} \ldots i_{n}}\left\langle\nu_{i} C_{i_{1} \ldots i_{n}}^{(n, n)} \nu_{i_{n+1}} \ldots \nu_{i_{n+\alpha}}\right\rangle .
$$

From equation (6), we can easily see that all the terms containing at least a symbol $\delta_{i_{q} i_{s}}$ with $1 \leq q, s \leq n$ give null results by contraction with $\mathbf{T}^{(n)}$. An exception is represented by the term containing the factor $D_{0}^{(n, n)} \nu_{i_{1}} \ldots \nu_{i_{n}}$ which, together with the factor $\nu_{i_{n+1}} \ldots \nu_{i_{n+\alpha}} \nu_{i}$, can give results different from zero. Since

$$
D_{0}^{(n, n)}=(-1)^{n}(2 n-1) ! !,
$$

the series (38) can be written as

$$
\begin{aligned}
\left\langle\boldsymbol{I}_{\sigma}\left(\mathbf{T}^{(n)}\right), \phi\right\rangle & =4 \pi \boldsymbol{e}_{i}(-1)^{n}(2 n-1) ! ! \lim _{\varepsilon \rightarrow 0} \sum_{\alpha=0}^{\infty} \frac{\varepsilon^{\alpha-n+1}}{\alpha !} \\
& \times \mathbf{T}_{i_{1} \ldots i_{n}}\left\langle\nu_{i_{1}} \ldots \nu_{i_{n}} \nu_{i_{n+1}} \ldots \nu_{i_{n+\alpha}} \nu_{i}\right\rangle\left(\partial_{i_{n+1}} \ldots \partial_{i_{n+\alpha}} \phi\right)_{0} .
\end{aligned}
$$

Firstly, we have to see what happens for negative powers of $\varepsilon$ in the terms of the series from the last equation. These negative powers are factors in the terms verifying the inequality $\alpha<n-1$. The expression is different from zero if and only if it contains some terms in which all the indices $i_{1} \ldots i_{n}$ are present as index pairs of Kronecker symbols $\delta_{i_{j} i_{k}}$ or $\delta_{i i_{q}}$ with $j, q=1, \ldots, n$ and $k \in\{n+1, \ldots, n+\alpha\}$. Otherwise, one obtains null values because of the contraction with an STF tensor. For $\alpha<n-1$ such terms cannot exist.

For $\alpha>n-1$, the corresponding terms from the series in equation (41) contain positive powers of $\varepsilon$. Consequently, the corresponding limits for $\varepsilon \rightarrow 0$ vanish. From this series only the term for which

$$
\alpha=n-1
$$

can be different from zero and the result of the limit in equation (41) is given by

$$
\begin{aligned}
\left\langle\boldsymbol{I}_{\sigma}\left(\mathbf{T}^{(n)}\right), \phi\right\rangle= & \frac{4 \pi(-1)^{n}(2 n-1) ! !}{(n-1) !} \\
& \quad \times \boldsymbol{e}_{i} \mathbf{T}_{i_{1} \ldots i_{n}}\left\langle\nu_{i_{1}} \ldots \nu_{i_{n}} \nu_{i_{n+1}} \ldots \nu_{i_{2 n-1}} \nu_{i}\right\rangle\left(\partial_{i_{n+1}} \ldots \partial_{i_{2 n-1}} \phi\right)_{0} \\
= & \frac{4 \pi(-1)^{n}(2 n-1) ! !}{(n-1) !}\left\langle\mathbf{T}^{(n)} \| \boldsymbol{\nu}^{2 n}\right\rangle \|\left(\boldsymbol{\nabla}^{n-1} \phi\right)_{0} .
\end{aligned}
$$

The corresponding $\delta$-function is therefore

$$
\boldsymbol{I}_{\sigma}\left(\mathbf{T}^{(n)}\right)=-\frac{4 \pi(2 n-1) ! !}{(n-1) !}\left\langle\mathbf{T}^{(n)} \| \boldsymbol{\nu}^{2 n}\right\rangle \| \nabla^{n-1} \delta(\boldsymbol{r}) .
$$

Let us consider the contraction

$$
\left\langle\mathbf{T}^{(n)} \| \boldsymbol{\nu}^{2 n}\right\rangle=\mathbf{T}_{i_{1} \ldots i_{n}}\left\langle\nu_{i_{1}} \ldots \nu_{i_{n}} \nu_{j_{1}} \ldots \nu_{j_{n}}\right\rangle .
$$

To this contraction contribute only the terms from the average of the $\nu$-product not containing factors $\delta_{i_{k} i l}$, with $1 \leq k, l \leq n$. According to equation (14), the terms giving non-zero contributions are of the form

$$
\frac{1}{(2 n+1) ! !} \delta_{i_{1} j_{1}} \ldots \delta_{i_{n} j_{n}}
$$


and all such terms are obtained considering the $n$ ! permutations of the indices $j_{1} \ldots j_{n}$ in this product. Therefore, the final expression in equation (44) is given by

$$
\boldsymbol{I}_{\sigma}\left(\mathbf{T}^{(n)}\right)=-\frac{4 \pi n}{2 n+1} \mathbf{T}^{(n)} \| \nabla^{n-1} \delta(\boldsymbol{r}) .
$$

Let us evaluate now the volume integral in equation (37):

$$
\begin{aligned}
& \left\langle J\left(\mathbf{T}^{(n)}\right), \boldsymbol{\nabla} \phi\right\rangle=\lim _{\varepsilon \rightarrow 0} \int_{\mathcal{D}_{\varepsilon}} \mathrm{d}^{3} x\left(\boldsymbol{\nabla}^{n} \| \frac{\mathbf{T}^{(n)}}{r}\right) \nabla \phi(\boldsymbol{r}) \\
& =\lim _{\varepsilon \rightarrow 0}\left[\oint_{\Sigma_{\varepsilon}} \mathrm{d} S \boldsymbol{\nu} \|\left(\boldsymbol{\nabla}^{n-1} \| \frac{\mathbf{T}^{(n)}}{r}\right) \boldsymbol{\nabla} \phi(\boldsymbol{r})-\int_{\mathcal{D} \varepsilon} \mathrm{d}^{3} x\left(\nabla^{n-1} \| \frac{\mathbf{T}^{(n)}}{r}\right) \boldsymbol{\nabla}^{2} \phi(\boldsymbol{r})\right] .
\end{aligned}
$$

The part corresponding to the surface integral can be written as

$$
\left\langle J_{\sigma}\left(\mathbf{T}^{(n)}\right), \boldsymbol{\nabla} \phi\right\rangle=\boldsymbol{e}_{i} \boldsymbol{\top}_{i_{1} \ldots i_{n}} \lim _{\varepsilon \rightarrow 0} \oint_{\Sigma_{\varepsilon}} \mathrm{d} S \nu_{i_{n}}\left(\partial_{i_{1}} \ldots \partial_{i_{n-1}} \frac{1}{r}\right) \partial_{i} \phi(\boldsymbol{r}) .
$$

Introducing the Taylor series for $\phi(\boldsymbol{r})$ and standing out the average over $\boldsymbol{\nu}$, we obtain

$$
\begin{aligned}
\left\langle J_{\sigma}\left(\mathbf{T}^{(n)}\right), \nabla \phi\right\rangle=4 \pi & \boldsymbol{e}_{i} \lim _{\varepsilon \rightarrow 0} \sum_{\alpha=0}^{\infty} \frac{\varepsilon^{\alpha-n+2}}{\alpha !} \mathbf{T}_{i_{1} \ldots i_{n}}\left\langle C_{i_{1} \ldots i_{n-1}}^{(n-1, n-1)} \nu_{i_{n}} \ldots \nu_{i_{n+\alpha}}\right\rangle \\
\times & \left(\partial_{i_{n+1}} \ldots \partial_{i_{n+\alpha}} \partial_{i} \phi\right)_{0} .
\end{aligned}
$$

Analogously to the reasoning from the previous case, we can see that the limit is zero since for $\alpha=n-2$

$$
\mathrm{T}_{i_{1} \ldots i_{n}}\left\langle C_{i_{1} \ldots i_{n-1}}^{(n-1, n-1)} \nu_{i_{n}} \nu_{i_{n+1}} \ldots \nu_{i_{2 n-2}}\right\rangle=0 .
$$

Therefore,

$$
\left\langle J\left(\mathbf{T}^{(n)}\right), \nabla \phi\right\rangle=-\lim _{\varepsilon \rightarrow 0} \int_{\mathcal{D}_{\varepsilon}} \mathrm{d}^{3} x\left(\nabla^{n-1} \| \frac{\mathbf{T}^{(n)}}{r}\right) \nabla^{2} \phi(\boldsymbol{r}),
$$

and by repeatedly applying the procedure, all terms cancel. Finally, only the surface integral from equation (37) gives a limit different from zero and

$$
\boldsymbol{I}\left(\mathbf{T}^{(n)}\right)=\boldsymbol{I}_{\sigma}\left(\mathbf{T}^{(n)}\right) \text {. }
$$

Introducing the result (45) in equation (27), we get

$$
\boldsymbol{E}_{(0)}^{(n)}=\frac{(-1)^{n}}{(n-1) !(2 n+1) \varepsilon_{0}} \mathcal{P}^{(n)} \| \nabla^{n-1} \delta(\boldsymbol{r}) .
$$

With this formula one obtains easily the results for $n=1$ and $n=2$. If we take the 3 -rd order term of the electric field, equation (50) gets the form:

$$
\boldsymbol{E}_{(0)}^{(3)}=-\frac{1}{14 \varepsilon_{0}} \mathcal{P}^{(3)} \| \nabla^{2} \delta(\boldsymbol{r})=-\frac{1}{14 \varepsilon_{0}} \boldsymbol{e}_{i} \mathcal{P}_{i j k} \partial_{j} \partial_{k} \delta(\boldsymbol{r})
$$

For the 4-th order multipolar term, equation (50) reads

$$
\boldsymbol{E}_{(0)}^{(4)}=\frac{1}{54 \varepsilon_{0}} \mathcal{P}^{(4)} \| \nabla^{3} \delta(\boldsymbol{r})
$$

and so on. 
Singular behaviour of the electromagnetic field

Concerning the magnetic field, selecting the singular point-like part of $\boldsymbol{B}^{(n)}$ from equation (28), we can write

$$
\begin{aligned}
\left\langle\boldsymbol{B}_{(0)}^{(n)}, \phi\right\rangle & =\frac{(-1)^{n-1} \mu_{0}}{4 \pi n !} \lim _{\varepsilon \rightarrow 0} \int_{\mathcal{D}_{\varepsilon}} \mathrm{d}^{3} x \mathcal{M}^{(n)} \|\left[\nabla^{n+1} \frac{1}{r}-\nabla^{n-1} \Delta \frac{1}{r}\right] \phi(\boldsymbol{r}) \\
& =\frac{(-1)^{n-1} \mu_{0}}{4 \pi n !} \lim _{\varepsilon \rightarrow 0} \int_{\mathcal{D}_{\varepsilon}} \mathrm{d}^{3} x \mathcal{M}^{(n)} \|\left[\nabla^{n+1} \frac{1}{r}+4 \pi\left(\boldsymbol{\nabla}^{n-1} \delta(\boldsymbol{r})\right)\right] \phi(\boldsymbol{r}) \\
& =\frac{(-1)^{n-1} \mu_{0}}{4 \pi n !} \lim _{\varepsilon \rightarrow 0} \int_{\mathcal{D}_{\varepsilon}} \mathrm{d}^{3} x\left(\mathcal{M}^{(n)} \| \nabla^{n+1} \frac{1}{r}\right) \phi(\boldsymbol{r})+\frac{\mu_{0}}{n !} \mathcal{M}^{(n)} \|\left(\boldsymbol{\nabla}^{n-1} \phi\right)_{0},
\end{aligned}
$$

since $\Delta(1 / r)=-4 \pi \delta(\boldsymbol{r})$. Written explicitly, this last equation becomes:

$$
\begin{gathered}
\left\langle\boldsymbol{B}_{(0)}^{(n)}, \phi\right\rangle=\frac{(-1)^{n-1} \mu_{0}}{4 \pi n !} \boldsymbol{e}_{i} \lim _{\varepsilon \rightarrow 0} \int_{\mathcal{D}_{\varepsilon}} \mathrm{d}^{3} x \mathcal{M}_{i_{1} \ldots i_{n}} \partial_{i}\left(\partial_{i_{1}} \ldots \partial_{i_{n}} \frac{1}{r}\right) \phi(\boldsymbol{r}) \\
+\frac{\mu_{0}}{n !} \boldsymbol{e}_{i} \mathcal{M}_{i_{1} \ldots i_{n-1} i}\left(\partial_{i_{1}} \ldots \partial_{i_{n-1}} \phi\right)_{0} .
\end{gathered}
$$

The integral on $\mathcal{D}_{\varepsilon}$ from equation (53) is of the same type as the integral from equation (36) and we can apply the result (45) obtained in the electric case, such that

$$
\boldsymbol{B}_{(0)}^{(n)}=\left(\frac{(-1)^{n} \mu_{0}}{(n-1) !(2 n+1)}+\frac{(-1)^{n-1} \mu_{0}}{n !}\right) \mathcal{M}^{(n)} \| \nabla^{n-1} \delta(\boldsymbol{r}),
$$

i.e.

$$
\boldsymbol{B}_{(0)}^{(n)}=\frac{(-1)^{n-1} \mu_{0}(n+1)}{n !(2 n+1)} \mathcal{M}^{(n)} \| \nabla^{n-1} \delta(\boldsymbol{r}) .
$$

Let us apply equation (54) for lower multipolar orders.

For the dipolar case:

$$
\boldsymbol{B}_{(0)}^{(1)}=\frac{2 \mu_{0}}{3} \boldsymbol{m} \delta(\boldsymbol{r}) .
$$

For $n=2$, the quadrupolar case,

$$
\boldsymbol{B}_{(0)}^{(2)}=-\frac{3 \mu_{0}}{10} \mathcal{M}^{(2)} \| \nabla \delta(\boldsymbol{r})
$$

For $n=3$,

$$
\boldsymbol{B}_{(0)}^{(3)}=\frac{2 \mu_{0}}{21} \mathcal{M}^{(3)} \| \nabla^{2} \delta(\boldsymbol{r}) .
$$

Before passing to the problem of the variable electromagnetic field, we point out some simple consequences of the formalism employed in the present section concerning the integrals over a spherical domain $\mathcal{D}_{R}$ of the electrostatic and magnetostatic fields, supposing the support of electric charges or currents included in $\mathcal{D}_{R}$.

Using equation (22) for representing the electric potential on the spherical surface $\Sigma_{R}$, we can write, employing the invariance of $\boldsymbol{E}$ to the substitutions (29), [1]

$$
\int_{\mathcal{D}_{R}} \mathrm{~d}^{3} x \boldsymbol{E}(\boldsymbol{r})=-\oint_{\Sigma_{R}} \mathrm{~d} S \boldsymbol{\nu} \Phi(\boldsymbol{r})=\frac{1}{4 \pi \varepsilon_{0}} \sum_{n \geq 1} \frac{(-1)^{n-1}}{n !} \oint_{\Sigma_{R}} \mathrm{~d} S \boldsymbol{\nu}\left(\boldsymbol{\nabla}^{n} \| \frac{\mathcal{P}^{(n)}}{r}\right) .
$$


By applying the Gauss theorem in the above integral, there were supposed fulfilled all the conditions of continuity of the field on the surface $\Sigma$ of the domain $\mathcal{D}$ where the electric charge is distributed.

Considering the surface integral corresponding to the $n$-th order,

$\boldsymbol{I}_{R}=\oint_{\Sigma_{R}} \mathrm{~d} S \boldsymbol{\nu}\left(\boldsymbol{\nabla}^{n} \| \frac{\mathcal{P}^{(n)}}{r}\right)=\boldsymbol{e}_{i} \oint_{\Sigma_{R}} \mathrm{~d} S \nu_{i} \partial_{i_{1}} \ldots \partial_{i_{n}} \frac{\mathcal{P}_{i_{1} \ldots i_{n}}}{r}=\frac{4 \pi}{R^{n-1}} \boldsymbol{e}_{i}\left\langle\nu_{i} C_{i_{1} \ldots i_{n}}^{(n, n)}\right\rangle \mathcal{P}_{i_{1} \ldots i_{n}}$.

Excepting the case $n=1$, the contractions of the angular averages with the $\mathbf{S T F}$ tensor $\mathcal{P}^{(n)}$ give null result such that

$$
\boldsymbol{I}_{R}=4 \pi \boldsymbol{e}_{i}\left\langle\nu_{i} \nu_{j}\right\rangle p_{j}=-\frac{4 \pi}{3} \boldsymbol{p}
$$

and

$$
\int_{\mathcal{D}_{R}} \mathrm{~d}^{3} x \boldsymbol{E}(\boldsymbol{r})=-\frac{1}{3 \varepsilon_{0}} \boldsymbol{p},
$$

i.e. equation (4.18) from Ref. [1].

In the case of the magnetic field, using the exterior solution for $\boldsymbol{A}$ given by equation (25), we can write

$$
\int_{\mathcal{D}_{R}} \mathrm{~d}^{3} x \boldsymbol{B}(\boldsymbol{r})=\oint_{\Sigma_{r}} \mathrm{~d} S \boldsymbol{\nu} \times \boldsymbol{A}=\frac{\mu_{0}}{4 \pi} \sum_{n \geq 1} \frac{(-1)^{n-1}}{n !} \oint_{\Sigma_{R}} \mathrm{~d} S \boldsymbol{\nu} \times\left[\boldsymbol{\nabla} \times\left(\boldsymbol{\nabla}^{n-1} \| \frac{\mathcal{M}^{(n)}}{r}\right)\right] .
$$

Considering the surface integral corresponding to the $n$-th order,

$$
\begin{aligned}
\boldsymbol{J}_{R} & =\boldsymbol{e}_{i} \varepsilon_{i j k} \varepsilon_{k l q} \oint_{\Sigma_{r}} \mathrm{~d} S \nu_{j}\left(\partial_{l} \partial_{i_{1}} \ldots \partial_{i_{n}} \frac{1}{r}\right) \mathcal{M}_{i_{1} \ldots i_{n}} \\
& =\boldsymbol{e}_{i} \varepsilon_{i j k} \varepsilon_{k l q} \oint_{\Sigma_{r}} \frac{\mathrm{d} \Omega(\boldsymbol{\nu})}{R^{n-1}} \nu_{j} C_{i_{1} \ldots i_{n-1} l}^{(n, n)} \mathcal{M}_{i_{1} \ldots i_{n-1} q} \\
& =\frac{4 \pi}{R^{n-1}} \boldsymbol{e}_{i} \varepsilon_{i j k} \varepsilon_{k l q}\left\langle\nu_{j} C_{i_{1} \ldots i_{n-1} l}^{(n, n)}\right\rangle \mathcal{M}_{i_{1} \ldots i_{n-1} q} .
\end{aligned}
$$

As in the electrostatic case, excepting the case $n=1$, the above contraction gives null result such that

$$
\boldsymbol{J}_{R}=-4 \pi \varepsilon_{i j k} \varepsilon_{k l q}\left\langle\nu_{j} \nu_{l}\right\rangle m_{q}=-\frac{4 \pi}{3}\left(\delta_{i l} \delta_{j q}-\delta_{i q} \delta_{j l}\right) \delta_{j l} m_{q}=\frac{8 \pi}{3} \boldsymbol{m}
$$

and consequently,

$$
\int_{\mathcal{D}_{R}} \mathrm{~d}^{3} x \boldsymbol{B}(\boldsymbol{r})=\frac{2 \mu_{0}}{3} \boldsymbol{m}
$$

i.e. equation (5.62) from Ref. [1].

\section{Singularities of the electromagnetic field: the dynamic case}

In the dynamic case, the multipole expansions of the potentials in an arbitrary point exterior to the domain $\mathcal{D}$ are given by the expressions [11]:

$$
\begin{aligned}
& \Phi(\boldsymbol{r}, t)=\frac{1}{4 \pi \varepsilon_{0}} \sum_{n \geq 1} \frac{(-1)^{n}}{n !} \nabla \| \frac{\mathbf{P}^{(n)}(\tau)}{r}, \\
& \boldsymbol{A}(\boldsymbol{r}, t)=\frac{\mu_{0}}{4 \pi} \sum_{n \geq 1} \frac{(-1)^{n-1}}{n !}\left[\nabla \times\left(\boldsymbol{\nabla}^{n-1} \frac{\mathbf{M}^{(n)}(\tau)}{r}\right)+\nabla^{n-1} \| \frac{\dot{\mathbf{P}}^{(n)}(\tau)}{r}\right] .
\end{aligned}
$$


As in the static case, for simplicity, we consider a neutral electric system $(Q=0)$. We write the multipole expansion of the electric field $\boldsymbol{E}(\boldsymbol{r}, t)$ derived from the potentials given in equation (58):

$$
\begin{aligned}
\boldsymbol{E}(\boldsymbol{r}, t)=- & \frac{1}{4 \pi \varepsilon_{0}} \sum_{n \geq 1} \frac{(-1)^{n}}{n !}\left\{\boldsymbol{\nabla}\left(\boldsymbol{\nabla}^{n} \| \frac{\mathbf{P}^{(n)}(\tau)}{r}\right)-\frac{1}{c^{2}} \boldsymbol{\nabla}^{n-1} \| \frac{\ddot{\mathbf{P}}^{(n)}(\tau)}{r}\right. \\
& \left.-\frac{1}{c^{2}} \boldsymbol{\nabla} \times\left[\boldsymbol{\nabla}^{n-1} \| \frac{\dot{\mathbf{M}}^{(n)}(\tau)}{r}\right]\right\} .
\end{aligned}
$$

The multipolar expansion of the magnetic field is given by

$$
\boldsymbol{B}(\boldsymbol{r}, t)=\frac{\mu_{0}}{4 \pi} \boldsymbol{\nabla} \times \sum_{n \geq 1} \frac{(-1)^{n-1}}{n !}\left[\boldsymbol{\nabla} \times\left(\boldsymbol{\nabla}^{n-1} \| \frac{\mathbf{M}^{(n)}(\tau)}{r}\right)+\boldsymbol{\nabla}^{n-1} \| \frac{\dot{\mathbf{P}}^{(n)}(\tau)}{r}\right] .
$$

Since

$$
\boldsymbol{\nabla} \times\left[\boldsymbol{\nabla} \times\left(\boldsymbol{\nabla}^{n-1} \| \frac{\mathbf{M}^{(n)}(\tau)}{r}\right)\right]=\boldsymbol{\nabla}\left(\boldsymbol{\nabla}^{n} \| \frac{\mathbf{M}^{(n)}(\tau)}{r}\right)-\Delta\left(\boldsymbol{\nabla}^{n-1} \| \frac{\mathbf{M}^{(n)}(\tau)}{r}\right),
$$

equation (60) can be written as

$$
\begin{aligned}
\boldsymbol{B}(\boldsymbol{r}, t) & =\frac{\mu_{0}}{4 \pi} \sum_{\geq 1} \frac{(-1)^{n-1}}{n !}\left[\boldsymbol{\nabla}\left(\boldsymbol{\nabla}^{n} \| \frac{\mathbf{M}^{(n)}(\tau)}{r}\right)-\Delta\left(\boldsymbol{\nabla}^{n-1} \| \frac{\mathbf{M}^{(n)}(\tau)}{r}\right)\right] \\
& +\frac{\mu_{0}}{4 \pi} \sum_{n \geq 1} \frac{(-1)^{n-1}}{n !} \boldsymbol{\nabla} \times\left(\boldsymbol{\nabla}^{n-1} \| \frac{\dot{\mathbf{P}}^{(n)}(\tau)}{r}\right) .
\end{aligned}
$$

Equations (59) and (61) represent the fields $\boldsymbol{E}$ and $\boldsymbol{B}$ for $r \neq 0$, where the multipole tensors considered at $t=\tau$ and divided by $r$ are solutions of the homogeneous wave equation. This property can be considered in any processing of the field multipole expansions. The corresponding delta-singularities, i.e. including the point $O$ in the domain of the field, will be expressed searching the extensions as generalized functions of the final expressions.

As in the static case, for higher orders of multipolar terms, $n \geq 2$, the labor involved in the calculation becomes prohibitive if we operate with the primitive moments $\mathbf{P}^{(n)}$ and $\mathbf{M}^{(n)}$. Fortunately, there is an invariance property of the electromagnetic field in the dynamic case, too, which allows us to replace in the expressions of $\boldsymbol{E}$ and $\boldsymbol{B}$ the tensors $\mathbf{P}^{(n)}$ and $\mathbf{M}^{(n)}$ by symmetric and trace-free (STF) tensors for any $n$ :

$$
\mathbf{P}^{(n)} \rightarrow \widetilde{\mathbf{P}}^{(n)}, \quad \mathbf{M}^{(n)} \rightarrow \widetilde{\mathbf{M}}^{(n)} .
$$

$\widetilde{\mathbf{P}}^{(n)}$ and $\widetilde{\mathbf{M}}^{(n)}$ are STF tensors (see Refs. [12, 13, 14] for $n \leq 3$ and [6, 11, 15] for arbitrary $n$ ). Theoretically, each such $\mathbf{S T F}$ tensor is an infinite series, but practically, we have to calculate only a finite number of terms from the multipole expansion such that the respective tensors are represented by finite sums. Consequently, we can operate in the following with the STF tensors : $\widetilde{\boldsymbol{p}}, \widetilde{\mathbf{P}}^{(2)}, \widetilde{\mathbf{P}}^{(3)}, \ldots$ for the electric cases, and $\widetilde{\boldsymbol{m}}, \widetilde{\mathbf{M}}^{(2)}, \ldots$ for the magnetic ones. However, for not invoking directly some results from the issues cited above, we introduce in Appendix B the lower orders STF tensors employed in the 
present paper, together with the introduction of the STF projections of the primitive momenta. In every case, we will establish what are the changes of different momentum tensors which compensate the effects of such substitutions.

Let us consider the problem of expressing the singularities of the electromagnetic field in $O$ when we are interested only in the contribution of the electric dipolar moment $\boldsymbol{p}(t)$. In this case we have an elementary charged system characterized only by the dipolar electric moment as, for example, the harmonic oscillator. Another case is when we are interested only in the first multipole approximation for a complex system, as an atom. For $n=1$, considering the contributions of the electric dipolar moment, equations (59) and (16) give

$$
\boldsymbol{E}_{(0)}^{(1, p)}(\boldsymbol{r}, t)=-\frac{1}{3 \varepsilon_{0}} \boldsymbol{p}(t) \delta(\boldsymbol{r}) .
$$

The singularity in the case of a magnetic dipole is

$\boldsymbol{B}_{(0)}^{(1, m)}=\frac{\mu_{0}}{4 \pi} \boldsymbol{e}_{i}\left[\partial_{i} \partial_{j} \frac{m_{j}(\tau)}{r}-\boldsymbol{e}_{i} \Delta \frac{m_{i}(\tau)}{r}\right]=\mu_{0} \boldsymbol{e}_{i}\left[-\frac{1}{3} m_{j}(t) \delta_{i j} \delta(\boldsymbol{r})+m_{i}(t) \delta(\boldsymbol{r})\right]$,

i.e.

$$
\boldsymbol{B}_{(0)}^{(1, m)}=\frac{2 \mu_{0}}{3} \boldsymbol{m}(t) \delta(\boldsymbol{r}) .
$$

Comparing equations (63) and (64) with equations (11) and (20) from the static case, one observes that the results for the dynamic case are obtained from the static one by the substitutions $\boldsymbol{p} \rightarrow \boldsymbol{p}(t), \boldsymbol{m} \rightarrow \boldsymbol{m}(t)$.

In equations (59) and (61), for the multipole expansions of the fields $\boldsymbol{E}$ and $\boldsymbol{B}$ we have a first general term of the form

$$
\nabla^{n+1} \| \frac{\mathbf{T}^{(n)}(\tau)}{r}=\boldsymbol{e}_{i} \partial_{i} \partial_{i_{1}} \ldots \partial_{i_{n}} \frac{\mathbf{T}_{i_{1} \ldots i_{n}}(\tau)}{r}
$$

introduced for $r \neq 0$ and with $\mathbf{T}^{(n)}$ a $\mathbf{S T F}$ tensor. Let us consider the extension of this function as a distribution isolating the point-like singularities by the definition

$$
\begin{aligned}
\left\langle\boldsymbol{I}\left(\mathbf{T}^{(n)}(t)\right), \phi\right\rangle & =\lim _{\varepsilon \rightarrow 0} \int_{\mathcal{D}_{\varepsilon}} \mathrm{d}^{3} x\left(\nabla^{n+1} \| \frac{\mathbf{T}^{(n)}(\tau)}{r}\right) \phi(\boldsymbol{r}) \\
& =\boldsymbol{e}_{i} \lim _{\varepsilon \rightarrow 0} \int_{\mathcal{D}_{\varepsilon}} \mathrm{d}^{3} x\left(\partial_{i} \partial_{i_{1}} \ldots \partial_{i_{n}} \frac{\mathbf{T}_{i_{1} \ldots i_{n}}(\tau)}{r}\right) \phi(\boldsymbol{r}) .
\end{aligned}
$$

The partial integration becomes

$$
\begin{aligned}
\left\langle\boldsymbol{I}\left(\mathbf{T}^{(n)}(t)\right), \phi\right\rangle=\lim _{\varepsilon \rightarrow 0} & {\left[\oint_{\Sigma_{\varepsilon}} \mathrm{d} S \boldsymbol{\nu}\left(\boldsymbol{\nabla}^{n} \| \frac{\mathbf{T}^{(n)}(\tau)}{r}\right) \phi(\boldsymbol{r})\right.} \\
& \left.-\int_{\mathcal{D}_{\varepsilon}} \mathrm{d}^{3} x\left(\boldsymbol{\nabla}^{n} \| \frac{\mathbf{T}^{(n)}(\tau)}{r}\right) \boldsymbol{\nabla} \phi(\boldsymbol{r})\right] .
\end{aligned}
$$

Considering only the surface integral from the last equation, employing equation (5) and the Taylor series of the function $\phi(\boldsymbol{r})$, after a regrouping of factors, we obtain

$$
\begin{aligned}
\left\langle\boldsymbol{I}_{\sigma}\left(\mathbf{T}^{(n)}(t)\right), \phi\right\rangle=\boldsymbol{e}_{i} & \lim _{\varepsilon \rightarrow 0} \oint_{\Sigma_{\varepsilon}} \mathrm{d} \Omega(\boldsymbol{\nu}) \sum_{l=0}^{n} \sum_{\alpha=0}^{\infty} \frac{\varepsilon^{\alpha-l+1}}{c^{n-l}} \\
& \times \nu_{i} C_{i_{1} \ldots i_{n}}^{(n, l)} \frac{\mathrm{d}^{n-l} \mathbf{T}_{i_{1} \ldots i_{n}}\left(\tau_{\varepsilon}\right)}{\mathrm{d} t^{n-l}} \nu_{i_{n+1}} \ldots \nu_{i_{n+\alpha}}\left(\partial_{i_{n+1}} \ldots \partial_{i_{n+\alpha}} \phi\right)_{0},
\end{aligned}
$$


Singular behaviour of the electromagnetic field

where $\tau_{\varepsilon}=r-\varepsilon / c$. As in the static case, we can write this last expression in terms of the averages upon the directions:

$$
\begin{aligned}
\left\langle\boldsymbol{I}_{\sigma}\left(\mathbf{T}^{(n)}(t)\right), \phi\right\rangle=4 \pi & \boldsymbol{e}_{i} \lim _{\varepsilon \rightarrow 0} \sum_{l=0}^{n} \sum_{\alpha=0}^{\infty} \frac{\varepsilon^{\alpha-l+1}}{c^{n-l} \alpha !} \frac{\mathrm{d}^{n-l}}{\mathrm{~d} t^{n-l}}\left\langle C_{i_{1} \ldots i_{n}}^{(n, l)} \mathrm{T}_{i_{1} \ldots i_{n}}\left(\tau_{\varepsilon}\right) \nu_{i_{n+1}} \ldots \nu_{i_{n+\alpha}} \nu_{i}\right\rangle \\
\times & \left(\partial_{i_{n+1}} \ldots \partial_{i_{n+\alpha}} \phi\right)_{0} .
\end{aligned}
$$

The general form of the coefficients $C^{(n, l)}$ is given by equation (6) and one can notice that only the term $D_{0}^{(n, l)} \nu_{i_{1}} \ldots \nu_{i_{n}}$ gives a non-zero contribution to the contraction with the $\mathbf{S T F}$ tensor $\mathbf{T}^{(n)}$. Therefore, we can write

$$
\begin{aligned}
\left\langle\boldsymbol{I}_{\sigma}\left(\mathbf{T}^{(n)}(t)\right)\right. & , \phi\rangle=4 \pi \boldsymbol{e}_{i} \lim _{\varepsilon \rightarrow 0} \sum_{l=0}^{n} \sum_{\alpha=0}^{\infty} \frac{\varepsilon^{\alpha-l+1}}{c^{n-l} \alpha !} D_{0}^{(n, l)} \\
& \times \frac{\mathrm{d}^{n-l}}{\mathrm{~d} t^{n-l}} \mathrm{~T}_{i_{1} \ldots i_{n}}\left(\tau_{\varepsilon}\right)\left\langle\nu_{i_{1}} \ldots \nu_{i_{n}} \nu_{i_{n+1}} \ldots \nu_{i_{n+\alpha}} \nu_{i}\right\rangle\left(\partial_{i_{n+1}} \ldots \partial_{i_{n+\alpha}} \phi\right)_{0}
\end{aligned}
$$

In this equation, the factor $\varepsilon^{\alpha-l+1}$ represents a negative power of $\varepsilon$ for $\alpha<l-1$. Since $l \leq n$, we can write

$$
\alpha<n-1 \text {. }
$$

In this case

$$
\left\langle\nu_{i_{1}} \ldots \nu_{i_{n}} \nu_{i_{n+1}} \ldots \nu_{i_{n+\alpha}} \nu_{i}\right\rangle \frac{\mathrm{d}^{n-l}}{\mathrm{~d} t^{n-l}} \mathrm{~T}_{i_{1} \ldots i_{n}}\left(\tau_{\varepsilon}\right)=0,
$$

since $n+\alpha+1<2 n$. Finally, the combinations of the factors $\varepsilon^{\alpha-l+1}$ for $\alpha<n-1$ with the terms from the Taylor series of $\mathrm{T}_{i_{1} \ldots i_{n}}\left(\tau_{\varepsilon}\right)$,

$$
\mathrm{T}_{i_{1} \ldots i_{n}}\left(\tau_{\varepsilon}\right)=\sum_{\lambda=0}^{\infty} \frac{(-1)^{\lambda} \varepsilon^{\lambda}}{\lambda ! c^{\lambda}} \frac{\mathrm{d}^{\lambda}}{\mathrm{d} t^{\lambda}} \mathrm{T}_{i_{1} \ldots i_{n}}(t)
$$

can give non negative powers of $\varepsilon$, but equation (171) remains valid.

The terms not containing $\varepsilon$ as factor verify the equality

$$
\alpha=l-1
$$

and they can give contribution different from zero.

For $\alpha>l-1$ the corresponding terms contain positive powers of $\varepsilon$ and have null limits for $\varepsilon \rightarrow 0$. Consequently, the sum over $\alpha$ is limited at $\alpha=l-1$.

Summarising, one can write the following expression for the limit in equation (66):

$$
\begin{aligned}
\left\langle\boldsymbol{I}_{\sigma}\left(\mathbf{T}^{(n)}(t)\right), \phi\right\rangle & =4 \pi \boldsymbol{e}_{i} \sum_{l=1}^{n} \sum_{\alpha=0}^{l-1} \frac{D_{(0)}^{(n, l)}}{c^{n-l} \alpha !} \\
& \times \frac{\mathrm{d}^{n-l}}{\mathrm{~d} t^{n-l}} \mathbf{T}_{i_{1} \ldots i_{n}}(t)\left\langle\nu_{i_{1}} \ldots \nu_{i_{n}} \nu_{i_{n+1}} \ldots \nu_{i_{n+\alpha}} \nu_{i}\right\rangle\left(\partial_{i n+1} \ldots \partial_{i_{n+\alpha}} \phi\right)_{0} .(73
\end{aligned}
$$

For $l<n$, equation (72) becomes $\alpha<n-1$ and equation (71) is verified; in the sum from equation (73) remain only the terms corresponding to $l=n$ and $\alpha=n-1$ which give:

$$
\begin{aligned}
\left\langle\boldsymbol{I}_{\sigma}\left(\mathbf{T}^{(n)}(t)\right), \phi\right\rangle & =\frac{(-1)^{n} 4 \pi(2 n-1) ! !}{(n-1) !} \\
& \times \mathbf{T}_{i_{1} \ldots i_{n}}(t)\left\langle\nu_{i_{1}} \ldots \nu_{i_{n}} \nu_{i_{n+1}} \ldots \nu_{i_{2 n-1}} \nu_{i}\right\rangle\left(\partial_{i_{n+1}} \ldots \partial_{i_{2 n-1}} \phi\right)_{0} .
\end{aligned}
$$


Comparing this result with the corresponding distribution (38) from the static case, one can see that equation (74) can be obtained from equation (43) by the substitution $\mathbf{T}^{(n)} \rightarrow \mathbf{T}^{(n)}(t)$.

The limit of the volume integral from equation (65) can be written as

$$
\begin{aligned}
& \left\langle J\left(\mathbf{T}^{(n)}\right), \boldsymbol{\nabla} \phi\right\rangle=\lim \int_{\mathcal{D}_{\varepsilon}} \mathrm{d}^{3} x\left(\boldsymbol{\nabla}^{n} \frac{\mathbf{T}^{(n)}(\tau)}{r}\right) \boldsymbol{\nabla} \phi(\boldsymbol{r}) \\
= & \lim _{\varepsilon \rightarrow 0}\left\{\oint_{\Sigma_{\varepsilon}} \mathrm{d} S\left[\boldsymbol{\nu} \cdot\left(\boldsymbol{\nabla}^{n-1} \| \frac{\mathbf{T}^{(n)}(\tau)}{r}\right)\right] \boldsymbol{\nabla} \phi(\boldsymbol{r})-\int_{\mathcal{D}_{\varepsilon}} \mathrm{d}^{3} x\left(\boldsymbol{\nabla}^{n-1} \| \frac{\mathbf{T}^{(n)}(\tau)}{r}\right) \| \boldsymbol{\nabla}^{2} \phi(\boldsymbol{r})\right\} .
\end{aligned}
$$

The limit of the surface integral from the last equation can be processed as in the case of equation (67), by writing

$$
\begin{aligned}
& \left\langle J_{\sigma}\left(\mathbf{T}^{(n)}\right), \boldsymbol{\nabla} \phi\right\rangle \\
= & \boldsymbol{e}_{i} \lim _{\varepsilon \rightarrow 0} \oint_{\Sigma_{\varepsilon}} \mathrm{d} S \nu_{i_{n}}\left(\partial_{i_{1}} \ldots \partial_{i_{n-1}} \frac{\mathrm{T}_{i_{1} \ldots i_{n}}(\tau)}{r}\right) \partial_{i} \phi(\boldsymbol{r})=\boldsymbol{e}_{i} \lim _{\varepsilon \rightarrow 0} 4 \pi \sum_{l=0}^{n-1} \sum_{\alpha=0}^{\infty} \frac{(-1)^{\lambda} \varepsilon^{\alpha-l+1}}{c^{n-l-1} \alpha !} \\
\times & \left\langle C_{i_{1} \ldots i_{n-1}}^{(n-1, n-1)} \nu_{i_{n}} \nu_{i_{n+1}} \ldots \nu_{i_{n+\alpha}}\right\rangle \frac{\mathrm{d}^{n-l-1}}{\mathrm{~d} t^{n-l-1}} \mathrm{~T}_{i_{1} \ldots i_{n}}\left(\tau_{\varepsilon}\right)\left(\partial_{i} \partial_{i_{n+1}} \ldots \partial_{i_{n+\alpha}} \phi\right)_{0} .
\end{aligned}
$$

Only the terms with $\alpha-l+1=0$ can give contributions different from zero but, since $l \leq n-1, \alpha$ verifies the inequality $\alpha \leq n-2$. Consequently, the average from the last equation contains at most a symbol $\delta_{i_{j} i_{k}}$ with $j, k \leq n$ and the contraction of this average with the $\mathbf{S T F}$ tensor $\mathbf{T}^{(n)}$ is zero. Finally, by reccurence, one can verify that the limit (75) is zero such that equation (74) is the result for the limit from equation (65).

Similar considerations lead to the conclusion that the second term, proportional to $\nabla^{n-1} \|\left(\ddot{\mathbf{P}}^{(n)}(\tau) / r\right)$, in the right parenthesis from the expression (59) of the electric field gives null contribution to the point-like singularities. The contribution of the magnetic moments to the point-like singularities of the electric field are defined by a distribution of the type

$$
\begin{array}{r}
\left\langle\boldsymbol{L}\left(\mathbf{T}^{(n)}\right), \phi\right\rangle=\boldsymbol{e}_{i} \lim _{\varepsilon \rightarrow 0} \varepsilon_{i j k} \int_{\mathcal{D}_{\varepsilon}} \mathrm{d}^{3} x\left(\partial_{j} \partial_{i_{1}} \ldots \partial_{i_{n-1}} \frac{\mathrm{T}_{i_{1} \ldots i_{n-1} k}(\tau)}{r}\right) \phi(\boldsymbol{r}) \\
=\boldsymbol{e}_{i} \lim _{\varepsilon \rightarrow 0}\left[\varepsilon_{i j k} \oint_{\Sigma_{\varepsilon}} \mathrm{d} S \nu_{j}\left(\partial_{i_{1}} \ldots \partial_{i_{n-1}} \frac{\mathrm{T}_{i_{1} \ldots i_{n-1} k}(\tau)}{r}\right) \phi(\boldsymbol{r})\right. \\
\left.\quad-\varepsilon_{i j k} \int_{\mathcal{D}_{\varepsilon}} \mathrm{d}^{3} x\left(\partial_{i_{1}} \ldots \partial_{i_{n-1}} \frac{\mathrm{T}_{i_{1} \ldots i_{n-1} k}(\tau)}{r}\right) \partial_{j} \phi(\boldsymbol{r})\right] .
\end{array}
$$

Concerning the surface integral from equation (77), introducing, as in previous cases, equation (5) for partial derivatives and the Taylor series for $\phi(\boldsymbol{r})$ (with the same notation for the various parameters), we notice that each term contains the contraction

$$
\varepsilon^{\alpha-l+1} \varepsilon_{i i_{n} k}\left\langle C_{i_{1} \ldots i_{n-1}}^{(n-1, n-1)} \nu_{i_{n}} \nu_{i_{n+1}} \ldots \nu_{i_{n+\alpha}}\right\rangle \mathrm{T}_{i_{1} \ldots i_{n-1} k}\left(\tau_{\varepsilon}\right) .
$$

This contraction can give contributions different from zero only for $\alpha=l-1 \leq n-2$. As one can easily see, for such values of $\alpha$ this contraction vanishes either because of the presence of the symbols $\delta_{i_{n} i_{q}}$ with $q \leq n-1$ or of $\delta_{i_{q} i_{s}}$ with $q, s \leq n-1$. The same 
conclusion applies to the contributions of the electric moments to the magnetic field as seen from equation (61).

The last type of singular distribution we have to search is for the term $\Delta\left[\nabla^{n-1} \|\left(\mathbf{T}^{(n)}(\tau) / r\right)\right]$ from the expansion (61) of the magnetic field. The corresponding point-like singular distribution can be obtained from the definition

$$
\begin{aligned}
\left\langle\boldsymbol{Q}\left(\mathbf{T}^{(n)}\right), \phi\right\rangle & =\boldsymbol{e}_{k} \lim _{\varepsilon \rightarrow 0} \int_{\mathcal{D}_{\varepsilon}} \mathrm{d}^{3} x \partial_{i_{1}} \ldots \partial_{i_{n-1}}\left(\Delta \frac{\mathrm{T}_{i_{1} \ldots i_{n-1} k}(\tau)}{r}\right) \phi(\boldsymbol{r}) \\
& =-4 \pi \lim _{\varepsilon \rightarrow 0} \int_{\mathcal{D}_{\varepsilon}} \mathrm{d}^{3} x \mathbf{T}_{i_{1} \ldots i_{n-1} k}(t) \phi(\boldsymbol{r}) \partial_{i_{1}} \ldots \partial_{i_{n-1}} \delta(\boldsymbol{r}) \\
& =-4 \pi \mathbf{T}_{i_{1} \ldots i_{n-1} k}(t)\left\langle\partial_{i_{1}} \ldots \partial_{i_{n-1}} \delta, \phi\right\rangle .
\end{aligned}
$$

Equations (74) and (78), inserted in equation (61), lead to the same conclusion as in the electric case: the distribution $\boldsymbol{B}_{(0)}^{(n)}$ in the dynamic case can be obtained from the static case, given by equation (54), with the substitution $\mathcal{M}^{(n)} \rightarrow \mathcal{M}^{(n)}(t)$.

\section{Conclusion}

Based on the results of the previous section, it is obvious that in the expansions (59) and (61) of $\boldsymbol{E}(\boldsymbol{r}, t)$ and $\boldsymbol{B}(\boldsymbol{r}, t)$, after performing the substitutions (62), only the terms

$$
\boldsymbol{\nabla}\left(\boldsymbol{\nabla}^{n} \| \frac{\widetilde{\mathbf{P}}^{(n)}(\tau)}{r}\right)
$$

for the electric field, and

$$
\boldsymbol{\nabla}\left(\boldsymbol{\nabla}^{n} \| \frac{\widetilde{\mathbf{M}}^{(n)}(\tau)}{r}\right), \quad \Delta\left(\boldsymbol{\nabla}^{n-1} \| \frac{\widetilde{\mathbf{M}}^{(n)}(\tau)}{r}\right)
$$

for the magnetic one give contributions to the $\delta$-singularities of the electromagnetic field. These contributions can be obtained by simply performing in the corresponding static expressions (501) and (54) of $\boldsymbol{E}$ and $\boldsymbol{B}$, the substitutions

$$
\mathcal{P}^{(n)} \rightarrow \widetilde{\mathbf{P}}^{(n)}(t), \quad \mathcal{M}^{(n)} \rightarrow \widetilde{\mathbf{M}}^{(n)}(t) .
$$

In Appendix B, the reduced STF moments for the first multipoles are established. Considering the substitutions (79) performed for all the multipoles, obviously $\widetilde{\mathbf{P}}^{(n)}(t)$ and $\widetilde{\mathbf{M}}^{(n)}(t)$ are actually represented by infinite series. Practically, as seen from this appendix, for a given multipole approximation, one deals only with finite sums. If, for example, we are interested only in the contributions to the singularities of the field from the electric octupole $(n=3)$ and magnetic quadrupole $(n=2)$, besides the contributions of the STF projections $\mathcal{P}^{(3)}$ and $\mathcal{M}^{(2)}$, contributions given by equations (51) and (56), we have to consider also a contribution of the type (31) of the electric dipolar toroidal moment $\boldsymbol{T}$ defined by equation (B.20). This last contribution is given by equation (31) with $\boldsymbol{p} \rightarrow \widetilde{\boldsymbol{p}}=\boldsymbol{p}-\dot{\boldsymbol{T}} / c^{2}$. If we are interested only in the contribution of the magnetic quadrupole, besides the contribution of the corresponding STF projection, we have to 
consider the contribution of an electric dipolar moment $\Delta^{\prime} \boldsymbol{p}$ given by equation (B.16), and so on.

We point out that there are alternative ways for introducing the moments $\widetilde{\mathbf{P}}$ and $\widetilde{\mathbf{M}}$. Obviously, the results from the present paper obtained through the formalism of the Cartesian tensors, can be obtained employing the expansions of the electromagnetic field in the formalism of the spherical tensors as in Refs. [1] and especially [12, 13]. One can employ the multipole moments derived by this formalism and then introduce the connection between spherical and Cartesian components of the multipoles [14.

The results of the present paper, if correct, could be useful in studying some problems of interest from the atomic, nuclear, elementary particles, and even gravitation physics.

\section{Appendix A. Expressions of the coefficients $C^{(n, l)}$}

The coefficients $C$ from equation (5) for $0 \leq n \leq 4$ are:

$$
\begin{aligned}
& C^{0,0}=1 ; \\
& C_{i}^{(1,0}=-\nu_{i}, \quad C_{i}^{1,1)}=-\nu_{i} ; \\
& C_{i j}^{(2,0)}=\nu_{i} \nu_{j}, \quad C_{i j}^{2,1}=3 \nu_{i} \nu_{j}-\delta_{i j}, \quad C_{i j}^{(2,2)}=3 \nu_{i} \nu_{j}-\delta_{i j} ; \\
& C_{i j k}^{(3,0)}=-\nu_{i} \nu_{j} \nu_{k}, \quad C_{i j k}^{(3,1}=-6 \nu_{i} \nu_{j} \nu_{k}+\delta_{\{i j} \nu_{k\}}, \\
& C^{(3,2)}=-15 \nu_{i} \nu_{j} \nu_{k}+3 \delta_{\{i j} \nu_{k\}}, \quad C^{(3,3)}=-15 \nu_{i} \nu_{j} \nu_{k}+3 \delta_{\{i j} \nu_{k\}} ; \\
& C_{i j k l}^{(4,0)}=\nu_{i} \nu_{j} \nu_{k} \nu_{l}, \\
& C_{i j k l}^{(4,1)}=10 \nu_{i} \nu_{j} \nu_{k} \nu_{l}-\delta_{\{i j} \nu_{k} \nu_{l\}}, \\
& C_{i j k l}^{(4,2)}=45 \nu_{i} \nu_{j} \nu_{k} \nu_{l}-6 \delta_{\{i j} \nu_{k} \nu_{l\}}+\delta_{\{i j} \delta_{k l\}}, \\
& C_{i j k l}^{(4,3)}=105 \nu_{i} \nu_{j} \nu_{k} \nu_{l}-15 \delta_{\{i j} \nu_{k} \nu_{l\}}+3 \delta_{\{i j} \delta_{k l\}}, \\
& C^{(4,4)}=105 \nu_{i} \nu_{j} \nu_{k} \nu_{l}-15 \delta_{\{i j} \nu_{k} \nu_{l\}}+3 \delta_{\{i j} \delta_{k l\}} .
\end{aligned}
$$

\section{Appendix B. Reduction of the multipole tensors}

Let us consider the electric quadripolar and magnetic dipolar contributions to the electromagnetic fields. The 4-polar electric moment $\mathbf{P}^{(2)}$ represents the first tensor for which one has to apply the substitution by the corresponding STF one. The tensor $\mathbf{P}^{(2)}$ is symmetric and the corresponding trace-free projection, denoted by $\mathcal{P}^{(2)}$ can be established as a combination of the form

$$
\mathcal{P}_{i j}=\mathrm{P}_{i j}-\Lambda \delta_{i j} .
$$

The parameter $\Lambda$ is determined from the condition $\mathcal{P}_{j j}=0$ :

$$
\Lambda=\frac{1}{3} \mathrm{P}_{i i} .
$$


Singular behaviour of the electromagnetic field

Firstly, we observe that $\boldsymbol{E}$ and $\boldsymbol{B}$ are not changed by the substitution $\mathbf{P}^{(2)} \rightarrow \boldsymbol{P}^{(2)}$. Indeed, equation (55) reads:

$$
\boldsymbol{E}(\boldsymbol{r}, t) \stackrel{\mathbf{P}^{(2)} \rightarrow \mathcal{P}^{(2)}}{\longrightarrow} \boldsymbol{E}(\boldsymbol{r}, t)+\frac{1}{8 \pi \varepsilon_{0}} \boldsymbol{e}_{i}\left[\Delta \partial_{i} \frac{\Lambda(\tau)}{r}-\frac{1}{c^{2}} \partial_{i} \frac{\ddot{\Lambda}(\tau)}{r}\right] .
$$

Since $r \neq 0$, equation (B.3) shows the invariance of the electric field to the considered substitution. Considering the change in the expression (157) by the substitution $\mathbf{P}^{(2)} \rightarrow \mathcal{P}^{(2)}$, we obtain

$$
\boldsymbol{B}(\boldsymbol{r}, t) \stackrel{\mathbf{P}^{(2)} \rightarrow \mathcal{P}^{(2)}}{\longrightarrow} \boldsymbol{B}(\boldsymbol{r}, t)+\frac{\mu_{0}}{8 \pi} \boldsymbol{e}_{i} \varepsilon_{i j k} \partial_{j} \partial_{l} \delta_{l k} \frac{\dot{\Lambda}(\tau)}{r}=\frac{\mu_{0}}{8 \pi} \boldsymbol{e}_{i} \varepsilon_{i j k} \partial_{j} \partial_{k} \frac{\dot{\Lambda}(\tau)}{r}=0,
$$

$\boldsymbol{B}$ being also invariant to this substitution. Let us consider the substitution

$$
\mathbf{P}^{(3)} \rightarrow \mathcal{P}^{(3)}
$$

in the multipole expansions of the fields $\boldsymbol{E}$ and $\boldsymbol{B}$. We have to express the components $\mathcal{P}_{i j k}$ in terms of the components $\mathrm{P}_{i j k}$. Obviously, the relations between the components of the tensors $\mathbf{P}^{(3)}$ and $\mathcal{P}^{(3)}$ are of the following form:

$$
\mathcal{P}_{i j k}=\mathrm{P}_{i j k}-\delta_{\{i j} \Lambda_{k\}}
$$

Considering the conditions on the traces $\mathcal{P}_{i i j}$ we write

$$
\mathcal{P}_{i i j}=\mathrm{P}_{i i j}-5 \Lambda_{j}=0,
$$

i.e.

$$
\Lambda_{i}=\frac{1}{5} \mathrm{P}_{i j j}=\frac{1}{5} \int_{\mathcal{D}} \mathrm{d}^{3} x r^{2} x_{i} \rho
$$

The effect of the substitution (B.5) in the expansion (55) of $\boldsymbol{E}$ is given by

$$
\begin{aligned}
\boldsymbol{E}_{p}^{(3)}(\boldsymbol{r}, t) & \rightarrow \boldsymbol{E}_{p}^{(3)}(\boldsymbol{r}, t)-\frac{1}{24 \pi \varepsilon_{0}} \boldsymbol{e}_{i}\left[\partial_{i} \partial_{j} \partial_{k} \partial_{l} \frac{\delta_{\{k l} \Lambda_{j\}}(\tau)}{r}-\frac{1}{c^{2}} \partial_{k} \partial_{l} \frac{\delta_{\{k l} \ddot{\Lambda}_{i\}}}{r}\right] \\
& =\boldsymbol{E}_{p}^{(3)}(\boldsymbol{r}, t)-\frac{1}{24 \pi \varepsilon_{0} c^{2}}\left[\boldsymbol{e}_{i} \partial_{i} \partial_{j} \frac{\ddot{\Lambda}_{j}(\tau)}{r}+\frac{1}{c^{2}} \boldsymbol{e}_{i} \frac{\ddot{\Lambda}_{i}(\tau)}{r}\right],
\end{aligned}
$$

or with the tensorial notation, the change of the field $\boldsymbol{E}$ is given by

$$
\boldsymbol{E}(\boldsymbol{r}, t) \stackrel{\mathbf{P}^{(3)} \rightarrow \mathcal{P}^{(3)}}{\longrightarrow} \boldsymbol{E}(\boldsymbol{r}, t)-\frac{1}{24 \pi \varepsilon_{0} c^{2}}\left[\boldsymbol{\nabla}\left(\boldsymbol{\nabla} \cdot \frac{\boldsymbol{\Lambda}(\tau)}{r}\right)-\frac{1}{c^{2}} \frac{\dddot{\boldsymbol{\Lambda}}(\tau)}{r}\right],
$$

where $\boldsymbol{\Lambda}=\boldsymbol{e}_{i} \Lambda_{i}$. This expression of the modification of $\boldsymbol{E}$ suggests us to try a change of the electric dipolar moment $\boldsymbol{p}$ by which the modification produced by the substitution (B.5) can be compensated. Let be the substitution:

$$
p \rightarrow p^{\prime}=p+\Delta p .
$$

We try to determine $\Delta \boldsymbol{p}$ such that the modification (35) of $\boldsymbol{E}$ is compensated. Since

$$
\begin{aligned}
\boldsymbol{E}(\boldsymbol{r}, t) & \stackrel{p \rightarrow \Delta \boldsymbol{p}}{\longrightarrow} \boldsymbol{E}(\boldsymbol{r}, t)+\frac{1}{4 \pi \varepsilon_{0}} \boldsymbol{e}_{i}\left[\partial_{i} \partial_{j} \frac{\Delta p_{j}(\tau)}{r}-\frac{1}{c^{2}} \frac{\Delta \ddot{p}_{j}(\tau)}{r}\right] \\
& =\boldsymbol{E}(\boldsymbol{r}, t)+\frac{1}{4 \pi \varepsilon_{0} c^{2}}\left[\boldsymbol{\nabla}\left(\boldsymbol{\nabla} \cdot \frac{\Delta \boldsymbol{p}(\tau)}{r}\right)-\frac{1}{c^{2}} \frac{\Delta \ddot{\boldsymbol{p}}}{r}\right],
\end{aligned}
$$


the comparison with equation (B.9) gives $\Delta \boldsymbol{p}=\ddot{\boldsymbol{\Lambda}} / 6 c^{2}$ and the substitution is in fact

$$
\boldsymbol{p} \rightarrow \boldsymbol{p}^{\prime}=\boldsymbol{p}+\frac{1}{6 c^{2}} \ddot{\boldsymbol{\Lambda}} .
$$

Let us consider also the change of $\boldsymbol{B}$ by the substitutions (B.5) and (B.12):

$$
\begin{aligned}
& \boldsymbol{B}(\boldsymbol{r}, t) \stackrel{\boldsymbol{p} \rightarrow \boldsymbol{p}^{\prime}, \mathbf{P}^{(3)} \rightarrow \mathcal{P}^{(3)}}{\longrightarrow} \boldsymbol{B}(\boldsymbol{r}, t)+\frac{\mu_{0}}{4 \pi} \boldsymbol{e}_{i} \varepsilon_{i j k} \partial_{j} \frac{\Delta \dot{p}_{k}(\tau)}{r}-\frac{\mu_{0}}{24 \pi} \boldsymbol{e}_{i} \varepsilon_{i j k} \partial_{j} \partial_{l} \partial_{q} \frac{\delta_{\{l q} \dot{\Lambda}_{k\}}(\tau)}{r} \\
= & \boldsymbol{B}(\boldsymbol{r}, t)+\frac{\mu_{0}}{24 \pi c^{2}} \boldsymbol{e}_{i} \varepsilon_{i j k} \partial_{j} \frac{\dddot{\Lambda}_{k}(\tau)}{r}-\frac{\mu_{0}}{24 \pi} \boldsymbol{e}_{i} \varepsilon_{i j k} \partial_{j} \partial_{l} \partial_{q} \frac{\left(\delta_{l q} \dot{\Lambda}_{k}(\tau)+\delta_{l k} \dot{\Lambda}_{q}(\tau)+\delta_{q k} \dot{\Lambda}_{l}(\tau)\right)}{r} \\
= & \boldsymbol{B}(\boldsymbol{r}, t)+\frac{\mu_{0}}{24 \pi c^{2}} \boldsymbol{e}_{i} \varepsilon_{i j k} \partial_{j} \frac{\dddot{\Lambda}_{k}(\tau)}{r}-\frac{\mu_{0}}{24 \pi} \boldsymbol{e}_{i} \varepsilon_{i j k} \partial_{j}\left(\Delta \frac{\dot{\Lambda}_{k}(\tau)}{r}+2 \partial_{k} \partial_{l} \frac{\dot{\Lambda}_{l}(\tau)}{r}\right)=0 .
\end{aligned}
$$

Since

$$
\Delta \frac{\dot{\Lambda}_{k}(\tau)}{r}=\frac{\dddot{\Lambda}_{k}(\tau)}{c^{2} r} \text { and } \varepsilon_{i j k} \partial_{j} \partial_{k}=0
$$

$\boldsymbol{B}$ is invariant to such a substitution. Concluding, we can substitute the tensor $\boldsymbol{P}^{(3)}$ by the STF tensor if we perform simultaneously the substitution $\boldsymbol{p} \rightarrow \boldsymbol{p}^{\prime}=\boldsymbol{p}+\ddot{\Lambda} / 6 c^{2}$.

Since we have to consider, together with the contributions of the electric octupolar moment to the $\delta$-singularities of the field, such contributions of the magnetic quadripolar moment also, we must show how one can substitute the momentum tensor $\mathbf{M}^{(2)}$ by the corresponding $\mathbf{S T F}$ projection $\mathcal{M}^{(2)}$. Let be the components

$$
\mathrm{M}_{i j}(t)=\frac{2}{3} \int_{\mathcal{D}} \mathrm{d}^{3} x x_{i}(\boldsymbol{r} \times \boldsymbol{J}(\boldsymbol{r}, t)) .
$$

Concerning the magnetic quadrupole moment $\mathrm{M}^{(2)}$, we have a simple procedure for obtaining the $\mathbf{S T F}$ projection (up to a factor). Let us write the identity:

$$
\mathrm{M}_{i j}=\frac{1}{2}\left(\mathrm{M}_{i j}+\mathrm{M}_{j i}\right)+\frac{1}{2}\left(\mathrm{M}_{i j}-\mathrm{M}_{j i}\right)
$$

where the first bracket represents the symmetric part of this tensor, and the second, the antisymmetric one. The symmetric part is, for this case $(n=2)$, a $\mathbf{S T F}$ tensor $\boldsymbol{M}^{(2)}$. Therefore,

$$
\mathrm{M}_{i j}=\mathcal{M}_{i j}+\frac{1}{2} \varepsilon_{i j k} \mathrm{~N}_{k}
$$

where

$$
\mathrm{N}_{k}=\varepsilon_{k i j} \mathrm{M}_{i j}=\frac{2}{3 \alpha} \int_{\mathcal{D}}[\boldsymbol{r} \times(\boldsymbol{r} \times \boldsymbol{J})]_{k} \mathrm{~d}^{3} x=\frac{2}{3 \alpha} \int_{\mathcal{D}}\left[(\boldsymbol{r} \cdot \boldsymbol{J}) x_{k}-r^{2} J_{k}\right] \mathrm{d}^{3} x .
$$

The substitution $\mathbf{M}^{(2)} \rightarrow \mathcal{M}^{(2)}$ in equation (55) gives:

$$
\begin{aligned}
& \boldsymbol{E}(\boldsymbol{r}, t) \stackrel{\mathbf{M}^{(2)}}{\rightarrow} \underset{\mathcal{M}^{(2)}}{ } \boldsymbol{E}(\boldsymbol{r}, t)-\frac{1}{16 \pi \varepsilon_{0} c^{2}} \boldsymbol{e}_{i} \varepsilon_{i j k} \varepsilon_{l k q} \partial_{j} \partial_{l} \frac{\dot{\mathbf{N}}_{q}(\tau)}{r} \\
& =\boldsymbol{E}(\boldsymbol{r}, t)+\frac{1}{16 \pi \varepsilon_{0} c^{2}}\left[\boldsymbol{\nabla}\left(\boldsymbol{\nabla} \cdot \frac{\dot{\boldsymbol{N}}(\tau)}{r}\right)-\Delta \frac{\dot{\boldsymbol{N}}(\tau)}{r}\right] \\
& =\boldsymbol{E}(\boldsymbol{r}, t)+\frac{1}{16 \pi \varepsilon_{0} c^{2}}\left[\boldsymbol{\nabla}\left(\boldsymbol{\nabla} \cdot \frac{\dot{\boldsymbol{N}}(\tau)}{r}\right)-\frac{\dddot{\boldsymbol{N}}(\tau)}{c^{2} r}\right] \text {, }
\end{aligned}
$$


where $\boldsymbol{N}=\mathrm{N}_{i} \boldsymbol{e}_{i}$. If we perform the substitution of the electric dipolar moment $\boldsymbol{p} \rightarrow \boldsymbol{p}^{\prime \prime}=\boldsymbol{p}+\Delta^{\prime} \boldsymbol{p}$, the electric field $\boldsymbol{E}$ is modified by an expression given by equation (B.11) (with $\Delta \rightarrow \Delta^{\prime}$ ). It is easy to see that the compensation of the changes in the electric field produced by the substitutions $\mathbf{M}^{(2)} \rightarrow \mathcal{M}^{(2)}$ and $\boldsymbol{p} \rightarrow \boldsymbol{p}^{\prime \prime}=\boldsymbol{p}+\Delta^{\prime} \boldsymbol{p}$, is given by the choice:

$$
\Delta^{\prime} \boldsymbol{p}=-\frac{1}{4 c^{2}} \dot{\boldsymbol{N}} .
$$

Concerning the change of $\boldsymbol{B}$ by the substitutions $\boldsymbol{p} \rightarrow \boldsymbol{p}+\Delta^{\prime} \boldsymbol{p}, \mathbf{M}^{(2)} \rightarrow \mathcal{M}^{(2)}$, we can write:

$$
\begin{aligned}
& \boldsymbol{B}(\boldsymbol{r}, t) \quad \stackrel{p \rightarrow \boldsymbol{p}+\Delta^{\prime} \boldsymbol{p}, \mathbf{M}^{(2)} \rightarrow \mathcal{M}^{(2)}}{\longrightarrow} \boldsymbol{B}(\boldsymbol{r}, t)+\frac{\mu_{0}}{4 \pi} \nabla \times \frac{\Delta^{\prime} \dot{\boldsymbol{p}}(\tau)}{r} \\
& +\frac{\mu_{0}}{16 \pi} \boldsymbol{e}_{i}\left[\varepsilon_{k j l} \partial_{i} \partial_{j} \partial_{k} \frac{\mathrm{N}_{l}(\tau)}{r}-\varepsilon_{k i l} \partial_{k} \Delta \frac{\mathrm{N}_{l}(\tau)}{r}\right] \\
& =\boldsymbol{B}(\boldsymbol{r}, t)-\frac{1}{16 \pi c^{2}} \boldsymbol{\nabla} \times \frac{\ddot{\boldsymbol{N}}(\tau)}{r}-\frac{\mu_{0}}{16 \pi}\left[\boldsymbol{\nabla}\left(\boldsymbol{\nabla} \cdot\left(\boldsymbol{\nabla} \times \frac{\boldsymbol{N}(\tau)}{r}\right)\right)-\frac{1}{c^{2}} \boldsymbol{\nabla} \times \frac{\ddot{\boldsymbol{N}}(\tau)}{r}\right] \\
& =\boldsymbol{B}(\boldsymbol{r}, t),
\end{aligned}
$$

i.e. the invariance of $\boldsymbol{B}$. Finally, we can express the total effect of the two substitutions $\mathbf{P}^{(3)} \rightarrow \mathcal{P}^{(3)}$ and $\mathbf{M}^{(2)} \rightarrow \mathcal{M}^{(2)}$ represented by the transformation of the electric dipolar moment:

or

$$
\boldsymbol{p} \stackrel{\mathbf{P}^{(3)} \rightarrow \mathcal{P}^{(3)}}{\boldsymbol{p}}+\frac{1}{6 c^{2}} \ddot{\boldsymbol{\Lambda}} \stackrel{\mathbf{M}^{(2)} \rightarrow \mathcal{M}^{(2)}}{\longrightarrow \boldsymbol{p}}=\boldsymbol{p}+\frac{1}{6 c^{2}} \ddot{\boldsymbol{\Lambda}}-\frac{1}{4 c^{2}} \dot{\boldsymbol{N}},
$$

$$
\boldsymbol{p} \stackrel{\mathbf{P}^{(3)} \rightarrow \mathcal{P}^{(3)}}{\longrightarrow} \mathbf{M}^{(3)} \rightarrow \mathcal{M}^{(3)} \widetilde{\boldsymbol{p}}=\boldsymbol{p}-\frac{1}{c^{2}} \dot{\boldsymbol{T}},
$$

where

$$
\boldsymbol{T}=\frac{1}{4} \boldsymbol{N}-\frac{1}{6} \dot{\Lambda}
$$

is the electric dipolar toroidal moment [12].

[1] J D Jackson, Classical Electrodynamics-2nd ed. (Wiley New York, 1975)

[2] C P Frahm, Am.J.Phys. 51, 826 (1983)

[3] P T Leung, G J Ni, Eur.J.Phys. 27, N1 (2006)

[4] W Weiglhofer, Am.J.Phys. 57, 455 (1989)

[5] P T Leung, Eur.J.Phys. 29, 137 (2008)

[6] T Damour, B R Iyer, Phys.Rev. D 43, 3259 (1991)

[7] K S Thorne, Rev.Mod.Phys. 52, 299 (1980)

[8] A Castellanos, M. Panizo, and J. Rivas, Am.J.Phys. 46, 1116 (1978)

[9] C Vrejoiu, St.Cerc.Fiz 36, 863 (1978) (in Romanian)

[10] H Gonzales, S R Juarez, P Kielanowski, M Loewe, Am.J.Phys. 66, 228 (1998)

[11] C Vrejoiu, J.Phys.A: Math.Gen. 35, 9911 (2002)

[12] V M Dubovik, A A Tscheshkov, Sov. J. Part. Nucl. 5, 318 (1974)

[13] V M Dubovik, V V Tugushev, Phys.Rep. 187, 145 (1990)

[14] E E Rădescu, G Vaman, Phys.Rev.E 65, 046609 (2002)

[15] C Vrejoiu, J.Phys.A: Math.Gen. 38, L505 (2005) 